\title{
NMR Based Quantum Information Processing: Achievements and Prospects
}

\author{
D. G. Cory ${ }^{1}$, R. Laflamme ${ }^{2}$, E. Knill ${ }^{2}$, L. Viola ${ }^{4}$, T. F. Havel ${ }^{3}$, N. Boulant ${ }^{1}$, \\ G. Boutis ${ }^{1}$, E. Fortunato ${ }^{1}$, S. Lloyd $^{4}$, R. Martinez ${ }^{2}$, C. Negrevergne ${ }^{2}$, \\ M. Pravia ${ }^{1}$, Y. Sharf ${ }^{1}$, G. Teklemariam ${ }^{5}$, Y. S. Weinstein ${ }^{4}$, W. H. Zurek ${ }^{2}$ \\ ${ }^{1}$ Dept. of Nuclear Engineering, MIT, Cambridge, MA 02139, USA \\ ${ }^{2}$ Los Alamos National Laboratory, Los Alamos, NM, 87545, USA \\ ${ }^{3}$ BCMP, Harvard Medical School, 240 Longwood Avenue, Boston, Massachusetts 02115 \\ ${ }^{4}$ Dept. of Mechanical Engineering, MIT, Cambridge, MA 02139, USA ${ }^{5}$ Dept. of Physics, MIT, Cambridge, MA 02139, USA
}

February 1, 2008

\begin{abstract}
Nuclear magnetic resonance (NMR) provides an experimental setting to explore physical implementations of quantum information processing (QIP). Here we introduce the basic background for understanding applications of NMR to QIP and explain their current successes, limitations and potential. NMR spectroscopy is well known for its wealth of diverse coherent manipulations of spin dynamics. Ideas and instrumentation from liquid state NMR spectroscopy have been used to experiment with QIP. This approach has carried the field to a complexity of about 10 qubits, a small number for quantum computation but large enough for observing and better understanding the complexity of the quantum world. While liquid state NMR is the only present-day technology about to reach this number of qubits, further increases in complexity will require new methods. We sketch one direction leading towards a scalable quantum computer using spin 1/2 particles. The next step of which is a solid state NMR-based QIP capable of reaching 10-30 qubits.
\end{abstract}

Corresponding author: David G. Cory

Massachusetts Institute of Technology

NW14-2217

150 Albany St

Cambridge, MA 02139

Phone: (617) 253-3806

fax: (617) 253-5405

dcory@mit.edu

\section{Contents}

\begin{tabular}{ll|}
\hline 1 & Introduction
\end{tabular}

2 Quantum simulation as a general approach to quantum information processors 4

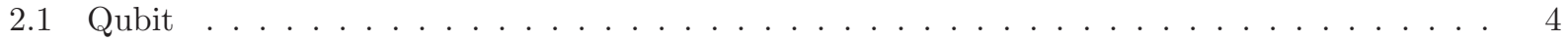

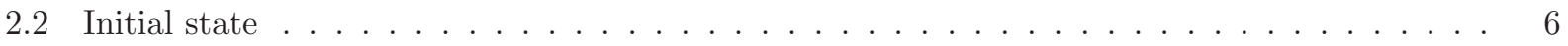

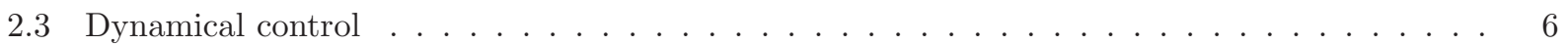

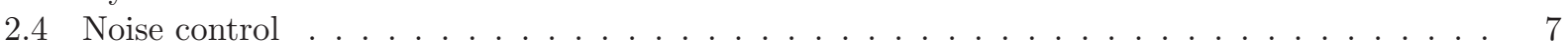

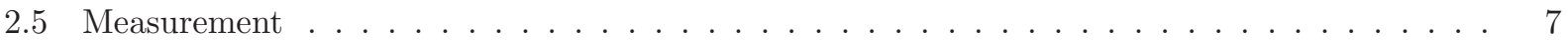


3 Introduction to liquid state NMR

3.1 Qubits in NMR . . . . . . . . . . . . . . . . . . . . . . . . . . . . 9

3.2 Hamiltonians . . . . . . . . . . . . . . . . . . . . . . . . . . . . . . . . . . 9

3.3 Unitary gates . . . . . . . . . . . . . . . . . . . . . . . . . . . . . . . 12

B.3.1 No-operation . . . . . . . . . . . . . . . . . . . . . . . . . . . 12

3.3.2 Control-not . . . . . . . . . . . . . . . . . . . . . . . . . . . . 13

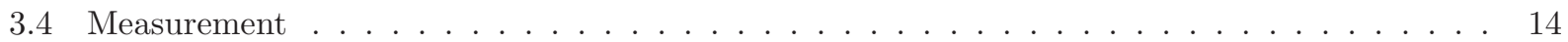

3.5 Non-unitary operations . . . . . . . . . . . . . . . . . . . . . . . . . 15

3.6 Pseudo-pure states . . . . . . . . . . . . . . . . . . . . . . . . . . . . . . . . . 15

3.7 Achievements of liquid state NMR QIP . . . . . . . . . . . . . . . . . . . . . . . . . . . . . . 17

4 Limitations of liquid state NMR QIP

5 Introduction to solid state NMR

$6 \quad$ Next generation QIP based on solid state NMR 21

6.1 General scheme . . . . . . . . . . . . . . . . . . . . . . . 21

6.2 Requirements . . . . . . . . . . . . . . . . . . . . . . . . . . 23

6.2.1 Scalable mapping . . . . . . . . . . . . . . . . . . . . . . . . . . . . . . 23

6.2 .2 State preparation . . . . . . . . . . . . . . . . . . . . . . . . . 24

6.2 .3 Gates . . . . . . . . . . . . . . . . . . . . . . . . . . . 24

6.2 .4 Noist . . . . . . . . . . . . . . . . . . . . . . . . . . . . . . . 24

6.2 .5 Measurement . . . . . . . . . . . . . . . . . . . . . . . 25

Future generations QIP based on engineered control of spin systems 25

8 Conclusion 2

\section{Introduction}

How can we turn quantum systems into useful and practical information processing devices? At present we do not know a detailed implementation but we have taken important steps both theoretically and experimentally to resolve this question. The ultimate such device will be a quantum computer (QC), and although such devices are beyond today's experimental reach, we do have related devices which are capable of performing information processing.

Quantum processors (QP) use the laws of quantum mechanics for computation, communication, and information storage [12, 31, 30, 76, 116, 13. What distinguishes quantum processors from classical ones is the ability to operate quantum mechanically on superpositions of quantum states and to exploit the resulting interference effects. To take advantage of QIP therefore requires quantum mechanically controllable physical devices. The difficulty of building suitable devices cannot be overestimated: the extreme fragility of quantum information compared to its classical counterpart makes robust manipulations much more difficult. Furthermore, it is exactly these fragile states that needs to be harnessed to achieve the potential of QIP. To turn QIP into a useful technology requires exploring vast, new territories of quantum mechanics to advance our understanding and our control of quantum mechanical systems [33]. Fortunately, as this volume shows, there is a rich variety of proposals for QIP implementations.

To evaluate, compare and measure the success of quantum devices for QIP requires milestones on the long road towards building a quantum computer. A QP is a physical device that operates via the laws of quantum mechanics, whose evolution can be adequately controlled, with noise and decoherence 138 held in check. Ultimately this control will lead to scalability, permitting fault tolerant computations such as Shor's factoring algorithm or quantum physics simulations. QPs can be compared on the basis of their controllability, reliability, scale and efficiency.

While all proposals for QIP devices include provisions for reliable control, to our knowledge scalability can only be achieved through the key theoretical discovery of fault tolerant quantum computing. Before this discovery, it was suggested that most computationally interesting problems would never be solved using 
quantum computers 68, 121: without methods of error control quantum computers lose their power. Fault tolerant quantum computing is based on the existence of quantum error correcting codes 107, 115. The main result is the accuracy threshold theorem [3, 59, 66, 93] which shows that errors in control can be tolerated: it is possible to efficiently compute as accurately as desired provided the basic device's precision exceeds a threshold. Consequently, errors of real devices are not a fundamental obstacle to QIP. The accuracy threshold depends on the types of noise that can affect a device and estimates for the maximum allowable error probabilities range from $10^{-6}$ to $10^{-3}$. Fidelity of control is perhaps the most useful benchmark for comparing QPs, being directly related to the scalability of quantum devices.

In this contribution we discuss current and proposed roles for nuclear magnetic resonance (NMR) based QIP, including liquid state, solid state and engineered systems (see also the contribution by Jones [56]). The relationship between information theory and NMR extends back to the beginning of NMR's experimental explorations. In 1946, the groups of Purcell [97] and Bloch [14] observed for the first time the magnetic induction of nuclear spins. This opened a new field of research leading to many important applications such as molecular structure determination, dynamics studies both in the liquid and solid state [37, and magnetic resonance imaging [78]. Perhaps the clearest example of early connections to information theory is the spin echo [49] where Hahn demonstrated that inhomogeneous interactions could be refocused to the extent that the phase of the nuclear spins retain information about the local field. This was followed by the magic echo in the solid state 101] which inverts the dipolar Hamiltonian so that the system can be returned to a prior state by using the fact that the history of spin evolution is stored in the density matrix. Indeed, the idea of using nuclear spins for information storage was suggested by Anderson and Hahn as early as 1955 [6, 局].

Recently, NMR was proposed as an accessible technology for QIP 74, 25, 28, 41. The NMR proposals are unique in that the first generation devices based on liquid state NMR have already manipulated systems of up to seven qubits using commercial technology. The main achievement of this technology has been to turn theoretical quantum algorithms into experimental ones. It is a technology where one can test the assumptions of the theory and where one can learn to deal with the imperfections of typical physical devices. The experiments have also inspired new theoretical investigations of QIP [61, 62, 63, 104, 91, 47.

Solid state NMR experiments provide a precedent for the controlled manipulation of very high order quantum coherences. For example, Pines and colleagues have demonstrated that systems of hundreds of spin $\frac{1}{2}$ particles can be coherently controlled [10]. In the solid state the decoherence rate can be reduced through coherent averaging and phase coherence times in the seconds have been achieved [27]. As the time for basic quantum operations is of the order of $100 \mu s$, the operational accuracy is in principle close to what is needed for scalable fault tolerance.

One of the key lessons of NMR spectroscopy over the last four decades and of NMR QIP over the last few years is that nuclear spin $\frac{1}{2}$ systems are extremely robust quantum systems. Their coherence can be precisely manipulated and they decohere slowly on the time scale of the qubit interactions. Our present efforts towards building a QP is based on nuclear spins, with key steps being:

- To develop liquid and solid state NMR methods for coherent control.

- To move to solid state NMR, developing methods for achieving high polarization.

- To use engineered systems with the goal of increasing clock rate and complexity.

This contribution is organized as follow. We introduce QIP, stressing the fact that QIP devices, which are first and foremost controllable quantum systems, emerge as an aspect of general principles of quantum simulation. The crucial elements required for understanding NMR QIP are introduced. These elements are dynamics, state initialization, and readout. We then explain the advantages and point out the limitations of the current NMR QIP prototypes based on the liquid state. To overcome these limitations, we propose a next generation of NMR QPs using solid state techniques. This generation will have very high polarization, faster gate times, slower decoherence rates, and will include resettable qubits to implement error correction. We conclude by reviewing what has been learned to date by NMR QIPs and give a brief discussion of the required steps for constructing scalable quantum computers. 


\section{Quantum simulation as a general approach to quantum infor- mation processors}

In the first chapter of this volume, DiVincenzo describes the five requirements for a general purpose, scalable, quantum computer, they can be summarized as follows:

\#1. A scalable mapping of the basic units of QIP, qubits, into physical systems.

\#2. The ability to initialize the state of the physical system to a known initial state corresponding via the mapping to a standard initial state of the qubits.

\#3. The availability of sufficient control via time-dependent Hamiltonians for implementing a universal set of quantum gates.

\#4. The availability of sufficient control of the relevant noise mechanism, allowing the implementation of fault tolerant QIP.

\#5. A qubit specific measurement capability.

This description assumes that QC are based on qubits and states. There is a more general description using observables and subsystems which is especially important for realizing fault tolerance in noisy quantum systems [65. Here we will conform to the state/qubit description but we believe that future QP will be based on an operator description, permitting the direct mapping of observables, and thereby the proper use of subsystems. When designing QPs it is helpful to consider the five requirements in the context of a general quantum simulation. Quantum simulation is best described by a diagram (Fig. 囵) which relates the abstract notion of a QP to its physical realization. In this diagram, the requirements correspond naturally to the crucial components, as will be explained shortly.

The goal of QIP is to transform an initial state $\rho^{s}$ by a series of quantum operations. It is implicitly assumed that the initial state is one that it is relatively easy to reach, such as a thermal state or the ground state so that we do not hide the difficulty of reaching a final state in the starting state. Once the final state is reached, we make a qubit specific measurement. We can restrict ourselves to measurements on a single qubit (e.g. qubit \#1) for deterministic algorithms. The process can be summarized by the following steps:

$$
\rho^{s} \stackrel{S_{m} \ldots S_{2} S_{1}}{\longrightarrow} \rho^{s}(t) \stackrel{\text { measure }}{\longrightarrow} \operatorname{Tr}\left\{\sigma_{z}^{1} \rho^{s}(t)\right\} .
$$

The task is to take a physical system and engineer it in such a way as to fashion a QP from its controlled dynamics. Any QP is thus first and foremost a physical system with its own internal and interaction Hamiltonians and modes of decoherence. This physical system is used to in effect simulate qubits, initial states, quantum gates, and measurements. The diagram of Fig. 1 shows the relationship between the ideal QP and the physical system in two steps (top to bottom), the first involving an ideal, noiseless physical system, and the second showing the process associated with the actual, noisy system. The correspondence between DiVincenzo's five requirements and the key steps in the simulation are described explicitly in the following subsections.

\subsection{Qubit}

The simplest building block for QIP is the qubit 105. A qubit is a two level system with basic states $|0\rangle$ and $|1\rangle$ corresponding to the configurations of a classical bit. Qubits can be represented by distinguishable spin $\frac{1}{2}$ particles to obtain a straightforward mapping from the computational to the physical space. When describing states of multi-qubits we will suppress the tensor product operator between them so that $\sigma_{z}^{1} \otimes \sigma_{z}^{2} \otimes \mathbb{1}^{3}$ will written as $\sigma_{z}^{1} \sigma_{z}^{2} \mathbb{1}^{3}$.

The states of the QP are related to states of the (ideal) physical system by the scalable mapping $\phi$ (Fig. 1). Scalability requires that the mapping can be efficiently extended to cover additional qubits. 


\section{Implementations of Quantum Algorithms}

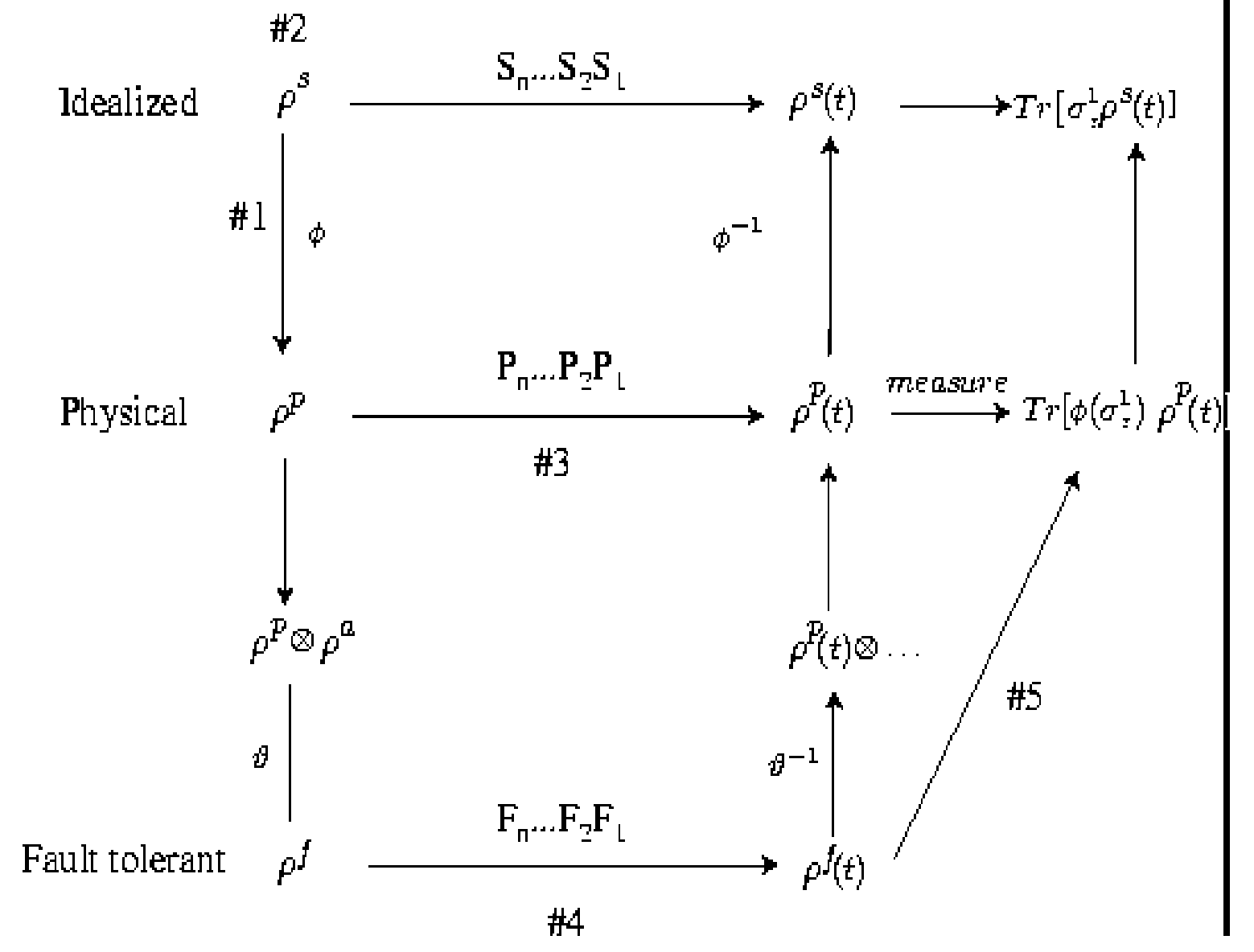

Figure 1: Diagrammatic representation of quantum simulation. The system to be simulated $(S)$, is mapped onto an ideal physical system $(P)$ whose evolution is made to mimic that of the simulated system by modulating the physical system's internal Hamiltonian using average Hamiltonian theory. The mapping is linear and invertible, and any Hamiltonian available in the physical space can be explored. The ideal physical system is implemented by a real physical system $\left(P^{\prime}\right)$ subject to noise. 


\subsection{Initial state}

The preparation of a known initial state is accomplished by preparing the state mapped by $\phi$ in the physical system,

$$
\rho^{p} \stackrel{\phi^{-1}}{\longrightarrow} \rho^{s}
$$

In most proposals for a $\mathrm{QC}$ requirement $\# 2$, preparing a known starting state, is achieved by cooling the system to the ground state either by contact to a cold reservoir or by active means. Examples of cooling methods in NMR include optical pumping [87] and dynamic nuclear polarization [132, 52]. An alternative approach is to map the initial state of the system into a state that behaves the same with respect to expectation value measurements up to an overall scale factor. Such states are called pseudo-pure states. They can be created from highly mixed states states as was shown early in the development of NMR QIP 117, 28, 118, 41. Sec. 3.6 gives a detailed implementation. Their use requires sufficiently high signalto-noise in the measurements which limits their scalability at high temperature. Nevertheless pseudo-pure states can be used to investigate and benchmark small QPs, and this is the approach taken in liquid state NMR. On the other hand, some efficient algorithms with no known efficient classical counterparts can be implemented with initial highly mixed states which are scalable 62 .

\subsection{Dynamical control}

In QIP we can think of applying control through a sequence of gates. The complete set of quantum gates are constructed by using time-varying Hamiltonians in the physical system to simulate the gates at each step. Thus the overall propagator in the ideal physical system is $V_{i}=\phi\left(U_{i}\right)$.

A method to analyze coherent control using time-varying Hamiltonians has been developed years ago (much before the advent of QIP) by Waugh and his colleagues [128, 48, 46 called average Hamiltonian theory (AHT). AHT provides a quantitative approach to understanding questions of coherent control and is complementary to the gate based methods of QIP. It enables one to solve for the evolution of the state at a time $T$ by writing the evolution of a time independent average Hamiltonian $\bar{H}(T)$. Average Hamiltonian theory separates the dynamics into a time invariant internal Hamiltonian, $H_{\text {int }}$, and a time dependent external Hamiltonian, $H_{\text {ext }}(t)$, giving a total Hamiltonian of $H_{\text {tot }}(t)=H_{\text {int }}+H_{\text {ext }}(t)$. The overall dynamics after a period of evolution is then given by

$$
U(T)=\mathcal{T} e^{-i \int_{0}^{T} d \tau H_{t o t}(\tau)}=e^{-i \bar{H} T}
$$

where $\mathcal{T}$ is the Dyson time ordering operator. The average Hamiltonian defined by this equation is the time independent Hamiltonian that would result in the same propagator if it were applied over the same period. If the total Hamiltonian commutes with itself at all times, $\bar{H}=\frac{1}{T} \int_{0}^{T} d \tau H_{t o t}(\tau)$. This is rarely the case. For sufficiently small $T$, the Magnus expansion [77, 131] provides a formal means of calculating the average Hamiltonian:

$$
\bar{H}=\bar{H}^{(0)}+\bar{H}^{(1)}+\bar{H}^{(2)}+\ldots
$$

where,

$$
\begin{aligned}
\bar{H}^{(0)} & =\frac{1}{T} \int_{0}^{T} d \tau H_{t o t}(\tau) \\
\bar{H}^{(1)} & =\frac{-i}{2 T} \int_{0}^{T} d \tau^{\prime \prime} \int_{0}^{\tau^{\prime}} d \tau^{\prime}\left[H_{t o t}\left(\tau^{\prime}\right), H_{t o t}\left(\tau^{\prime \prime}\right)\right] .
\end{aligned}
$$

Examples of the use of AHT to implement quantum gates are included in the following section.

The development of AHT is a prime example of how the development of NMR anticipated many of the ideas of QIP using a different language. In the context of NMR Waugh demonstrated that:

- AHT can be used to design a control scheme for implementing a desired average Hamiltonian,

- Any average Hamiltonian (up to a scalar multiple) can be implemented in most NMR systems of distinguishable spins 43 . 
- The average Hamiltonian can be implemented to an arbitrary precision if decoherence (random noise in the system and in the control) is neglected.

AHT solves the problem of making available a complete set of gates in the physical systems to meet requirement \#3. The nuclear spins in a molecule can be chosen to be coupled by internal Hamiltonians in a connected coupling network (explained in Sec. B), and the results of AHT given above show that in this case, a complete set of gates can be implemented in principle.

There are many equivalent sets of gates that are complete for the purpose of QIP. There are minimal sets consisting of one gate only, but for efficient physical implementation, other sets are more convenient [9, 32, 75 . The basic property of these gate sets are that any unitary transformation can in principle be decomposed to arbitrary precision into a sequence of gates from the set!. One such set consists of the operations on one and two qubits given in the next two equations,

$$
U(\mathbf{v} \phi)=\exp \left(-i\left(v_{x} \sigma_{x}+v_{y} \sigma_{y}+v_{z} \sigma_{z}\right) \phi / 2\right),
$$

where $\mathbf{v}=\left(v_{x}, v_{y}, v_{z}\right)$ is a real unit vector. This defines a rotation by $\phi$ around $\mathbf{v}$ in the Bloch (or Poincaré) sphere associated with the state space of a qubit. The standard two qubit operation is the controlled-not defined by

$$
U_{\text {cnot } 12}=|0\rangle\left\langle 0\left|\mathbb{1}^{2}+\right| 1\right\rangle\langle 1| \sigma_{x}^{2} .
$$

It flips the second qubit only if the first bit is in the state $|1\rangle$. The gates in this set can be applied to any one or two qubits in the computational space. Example NMR implementations of these gates are given in Sec. 3 .

The extent to which requirement \#3 is met in NMR depends on the details of the system used. Since, as explained above, the control is in principle sufficient to implement the desired gates with arbitrarily accuracy, it is the extent to which random noise affects the gates that is important. Probabilities of errors per gate due to random noise can be as low as .01 in liquid state systems for the most easily implementable two qubit gates.

\subsection{Noise control}

Noise can be controlled in principle through quantum error correction. In order that fault tolerant method be effective it is sufficient to be below an accuracy threshold, i.e. that the noise per gate be smaller than a threshold value. The threshold is determined by the the types of errors and control we have over the device. In order to implement these fault tolerant procedures we have to increase the size of the physical system but only by a "reasonable" amount (polynomial in the size of th problem considered).

\subsection{Measurement}

The measurement of one or more qubits translates via $\phi$ to a corresponding measurement in the physical system. Usually the measurement is required to be projective. However, for most purposes, it is sufficient to make a noisy expectation value measurement of a single qubit (this works for all computations where a specific answer is desired 137). Projective measurements with known outcomes are in fact useful only for improvements in efficiency. Thus we require that it is possible to measure $\operatorname{tr}\left[\sigma_{z}{ }^{1} \rho^{s}(t)\right]$ with constant signal to noise. If it were possible to measure this with infinite signal to noise, i.e. exactly, then an apparently much more powerful model of computation is obtained, with simple algorithms for solving NP hard problems. The quantity measured corresponds to $\operatorname{tr}\left[\phi\left(\sigma_{z}{ }^{1}\right) \rho^{p}(t)\right]$ in the physical system.

The ability to make expectation value measurements as demanded by our version of requirement \#5 is easily available in NMR and is what makes NMR such a useful technology.

\section{Introduction to liquid state NMR}

NMR provides a valuable test bed for QIP ideas, since both the internal Hamiltonian and the relaxation superoperator are well known and reliable methods for controlling the dynamics are available. No other

\footnotetext{
${ }^{1}$ Almost all unitary transformations require exponentially many gates to implement 9,60 .
} 


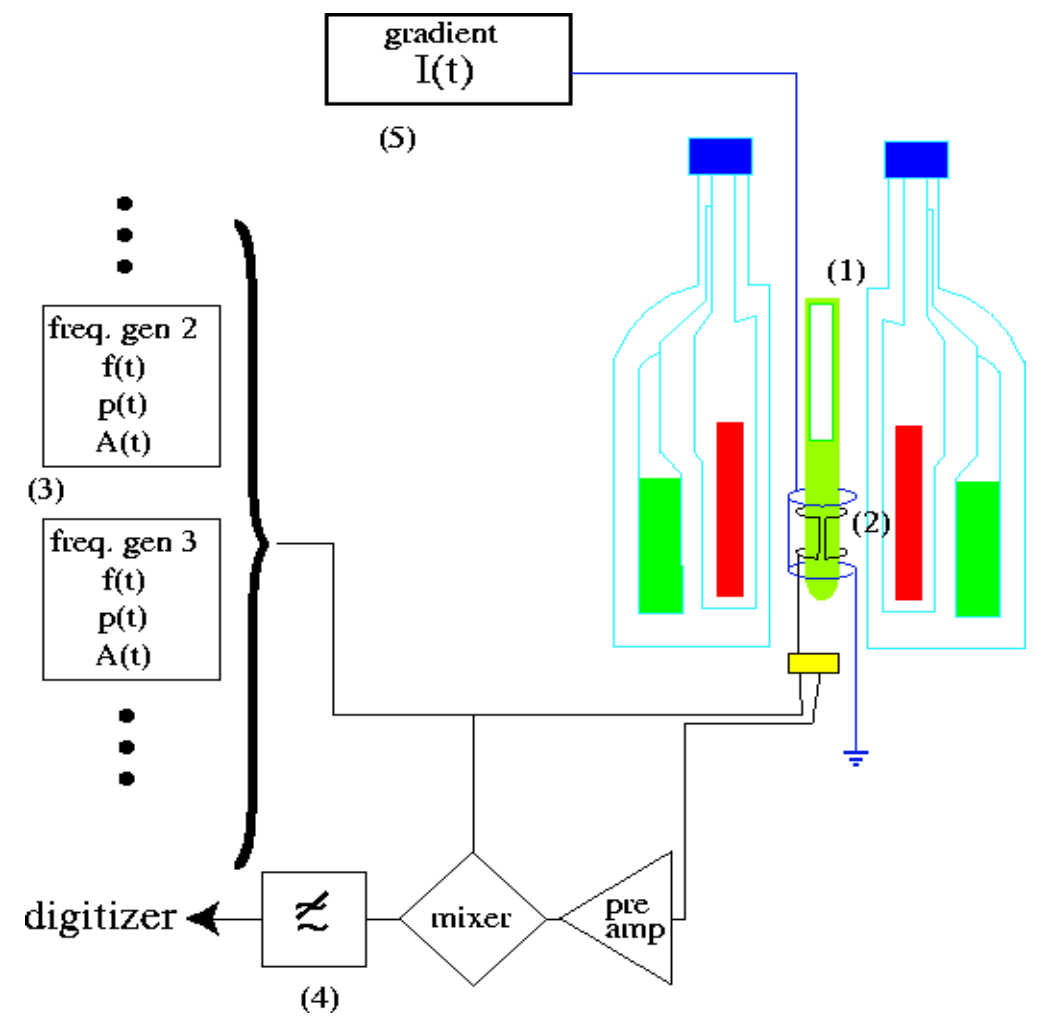

Figure 2: A schematic block diagram of the key components of an NMR system. The sample (1) is placed in a strong, homogeneous magnetic field (typically a superconducting magnet of 8-14 T) inside a RF cavity (2) tuned to the resonance frequencies of the nuclei of interest (typically ${ }^{1} H$ and ${ }^{13} \mathrm{C}$ ). At a field of $9.4 \mathrm{~T}$, this corresponds to resonance frequencies of 400 and $100 \mathrm{MHz}$ respectively. A parallel set of computer-controlled $\mathrm{RF}$ transmitters (3) are connected to the cavity with the frequency, phase, and amplitude of each channel independently controllable. The same RF cavity is used for detection, typically of only one of the nuclear species $\left({ }^{1} \mathrm{H}\right.$ or $\left.{ }^{13} \mathrm{C}\right)$. The detected signal (4) is referenced to the transmitter frequency, so only the difference frequencies are observed. Most modern NMR systems are also provided with a programmable magnetic field gradient unit (5) which is a convenient means for artificially introducing decoherence.

technology has so far reached the ability to control the number of qubits accessible in NMR (currently seven). In addition, there are forty years of experience in using NMR to explore chemical structure, dynamics and reactions. Such experience has led to robust instrumentation capable of complex experiments and to a wealth of practical methods for understanding spin dynamics.

A block diagram of an NMR spectrometer for QIP is shown in Fig. 2. Even though the goals of standard NMR experiments and QIP are quite different, the instrumentation requirements are very similar and the same device can be used for both purposes. In liquid state NMR spectroscopy the goal is to characterize the chemistry/dynamics of a solution containing known or unknown molecules via a series of well characterized experiments tailored to elicit specific components of the Hamiltonian or relaxation superoperator in an easy to interpret fashion. In NMR QIP the goal is to characterize the effective overall transformation of a complex series of operations on a very well characterized sample whose internal Hamiltonian and relaxation superoperator are precisely known. Ultimately the goal is to apply series of transformations sufficiently complex to be beyond the capacity of a classical computer. 


\subsection{Qubits in NMR}

In NMR, the qubits are spin $\frac{1}{2}$ nuclei in a molecule. Although in principle other nuclei can be used by observing that a spin $m$ nucleus can represent $\log _{2}(2 m+1)$ qubits, this however increases the complexity of implementing the standard quantum gates given the Hamiltonians that are usually available for control.

Distinguishable spin $\frac{1}{2}$ particles are convenient physical qubits since, being two state systems, the mapping from the computational space is usually straightforward and consists of associating each qubit with one of the spins. For the liquid and solid state NMR devices the spins are distinguishable on the basis of the internal Hamiltonian which is given by the chemistry of the sample. For engineered systems it is assumed that nuclear spins and thus qubits can be spatially localized.

The robustness of spin $\frac{1}{2}$ particles is due to the fact that they can interact directly only with magnetic, not with electric fields. This is particularly valuable since other than adjacent qubits, sources of magnetic fields, will generally be distant and as a result the interaction takes on a dipole-dipole symmetry. Nuclear spins have the additional advantage of being shielded by the electron cloud and thus being very well isolated from most sources of fluctuating magnetic fields, leading typically to long decoherence times.

\subsection{Hamiltonians}

NMR relies on a strong applied magnetic field to provide the dominant terms of the internal Hamiltonian. The Zeeman interaction then provides a convenient and uniform axis of quantization, with the sum of the $\sigma_{z}$ giving useful quantum numbers. As a result, in the weak coupling limit (i.e when the difference between chemical shifts is much larger than the coupling between spins), the eigenstructure of the internal Hamiltonian is easy to determine. The form of the internal Hamiltonian of a molecule's nuclear spins is then to very good approximation,

$$
H=\frac{1}{2} \sum_{i} \omega_{i} \sigma_{z}^{i}+\frac{\pi}{2} \sum_{i \neq j} J_{i j} \sigma_{z}^{i} \sigma_{z}^{j}
$$

where the summation is over the nuclear spins. For liquid state NMR a typical sample contains $10^{18}$ molecules, each one acting as an independent QP. Many copies are necessary since the Zeeman interaction is very weak and approximately $10^{15}$ spins are necessary to observe a signal. The spectrometer records the average state of the spins and the computation has to preserve the Hilbert space spanned by a single molecule. The through space, spin-spin, dipolar interaction between molecules is averaged to zero by random molecular motions on the time scale of the control and measurement normally used, and is therefore only indirectly observable via the effect on decoherence.

The form of the Hamiltonian is convenient for QIP. The single particle terms are naturally useful to distinguish qubits, while the two-particle terms form the building blocks of conditional, two qubit gates. The constants $\omega_{i}$ and $J_{i j}$ depend on the structure and electron configuration of the molecule. The resonance frequencies $\omega_{i}$ depend on the efficiency of screening of the nuclear spins from the applied magnetic field by the surrounding electrons and the scalar couplings $J_{i j}$ are mediated by electrons in molecular orbitals that overlap both nuclear spins.

The constants of the internal Hamiltonian of the nuclear spins of a molecule can be derived from an NMR spectrum acquired following a non-selective RF excitation pulse. Fig. 3 shows the spectrum for ${ }^{13} \mathrm{C}$ labeled alanine. (The ${ }^{1} H$ 's were removed from the Hamiltonian (and spectrum) through spin decoupling, described in Sec. 3.3.1.) The constants can be read off (in frequency units) from the positions of the peaks. Thus the three ${ }^{13} \mathrm{C}$ nuclei at $9.4 \mathrm{~T}$ are governed by the Hamiltonian

$$
\begin{aligned}
H_{\text {alanine }}= & \pi\left[10^{8}\left(\sigma_{z}^{a}+\sigma_{z}^{b}+\sigma_{z}^{c}\right)-12580 \sigma_{z}^{b}+3440 \sigma_{z}^{c}\right]+ \\
& (\pi / 2)\left[53 \sigma_{z}^{a} \sigma_{z}^{b}+38 \sigma_{z}^{a} \sigma_{z}^{c}+1.2 \sigma_{z}^{b} \sigma_{z}^{c}\right],
\end{aligned}
$$

where all of the frequencies are given in Hz. The last two terms of the first line induce so-called chemical shifts which distinguish qubits. The second line gives the couplings between qubits.

The simplicity of the internal Hamiltonian also carries over to the allowed modes of decoherence since there is no energy change associated with a phase shift about the applied external field. The interaction of the environment thus only attenuates the off diagonal elements of the density matrix (it does not mix these), and the Liouvillian has a simple and sparse form. The relaxation superoperator is simplest to describe in 


\section{Spectrum of alanine}

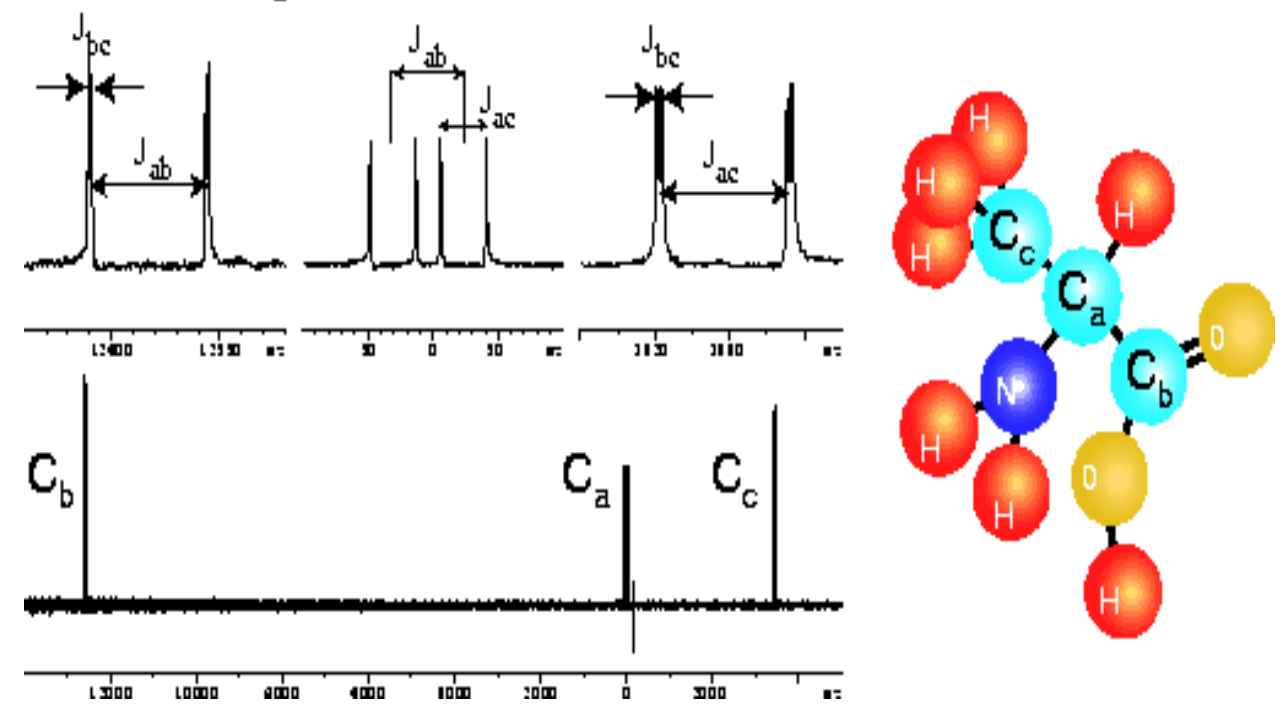

Figure 3: ${ }^{13} \mathrm{C}$ proton-decoupled NMR spectrum of alanine at $9.4 \mathrm{~T}$ showing the chemical shift differences between the three chemically distinct carbon spins, and the couplings between them. The width of the resonances provides an upper bound on the decoherence rates. Note that in the expanded views of the coupling between spins the horizontal scales are not uniform.

terms of a set of product operators in the raising and lowering basis of the quantization axis. The state of the system at any time on the other hand can be described as a sum of direct products of the states of the individual spins, and these product states have very simple transformation rules under both the internal and external Hamiltonians 114, 122, 90].

In NMR the relaxation superoperator [99, 100] is called the Redfield kite, named after Alfred Redfield who first described it. It is called a kite to provide a descriptive name for the structure of the non-zero elements. For a three spin system, the superoperator has $64^{2}$ elements, of which only 120 are non-zero in the absence of cross-correlation. The form of the relaxation superoperator is summarized compactly in the following table.

\begin{tabular}{l|ccccccccccccc} 
& $\mathbb{1}$ & $\sigma_{z}$ & $\sigma_{z}$ & $\sigma_{z}$ & $\sigma_{ \pm}$ & $\sigma_{ \pm}$ & $\sigma_{ \pm}$ & $\sigma_{ \pm}$ & $\sigma_{ \pm}$ & $\sigma_{ \pm}$ & $\sigma_{ \pm}$ & $\sigma_{ \pm}$ & $\sigma_{ \pm}$ \\
& $\mathbb{1}$ & $\mathbb{1}$ & $\sigma_{z}$ & $\sigma_{z}$ & $\mathbb{1}$ & $\sigma_{z}$ & $\sigma_{z}$ & $\sigma_{ \pm}$ & $\sigma_{ \pm}$ & $\sigma_{ \pm}$ & $\sigma_{ \pm}$ & $\sigma_{ \pm}$ & $\sigma_{ \pm}$ \\
& $\mathbb{1}$ & $\mathbb{1}$ & $\mathbb{1}$ & $\sigma_{z}$ & $\mathbb{1}$ & $\mathbb{1}$ & $\sigma_{z}$ & $\mathbb{1}$ & $\sigma_{z}$ & $\mathbb{1}$ & $\sigma_{z}$ & $\sigma_{ \pm}$ & $\sigma_{ \pm}$ \\
\hline $11111: 1$ & $\mathrm{X}$ & $\mathrm{X}$ & $\mathrm{X}$ & $\mathrm{X}$ & & & & & & & & & \\
$\sigma_{z} \mathbb{1 1 1}: 3$ & $\mathrm{X}$ & $\mathrm{X}$ & $\mathrm{X}$ & $\mathrm{X}$ & & & & & & & & \\
$\sigma_{z} \sigma_{z} \mathbb{1 1}: 3$ & $\mathrm{X}$ & $\mathrm{X}$ & $\mathrm{X}$ & $\mathrm{X}$ & & & & & & & & \\
$\sigma_{z} \sigma_{z} \sigma_{z}: 1$ & $\mathrm{X}$ & $\mathrm{X}$ & $\mathrm{X}$ & $\mathrm{X}$ & & & & & & & & \\
$\sigma_{ \pm} 111: 6$ & & & & & $\mathrm{X}$ & & & & & & & \\
$\sigma_{ \pm} \sigma_{z} \mathbb{1 1}: 12$ & & & & & & $\mathrm{X}$ & & & & & & \\
$\sigma_{ \pm} \sigma_{z} \sigma_{z}: 6$ & & & & & & & $\mathrm{X}$ & & & & & \\
$\sigma_{ \pm} \sigma_{\mp} \mathbb{1 1}: 6$ & & & & & & & & $\mathrm{X}$ & & & & \\
$\sigma_{ \pm} \sigma_{\mp} \sigma_{z}: 6$ & & & & & & & & & $\mathrm{X}$ & & & \\
$\sigma_{ \pm} \sigma_{ \pm} \mathbb{1}: 6$ & & & & & & & & & & $\mathrm{X}$ & & & \\
$\sigma_{ \pm} \sigma_{ \pm} \sigma_{z}: 6$ & & & & & & & & & & & $\mathrm{X}$ & & \\
$\sigma_{ \pm} \sigma_{ \pm} \sigma_{\mp}: 6$ & & & & & & & & & & & $\mathrm{X}$ & \\
$\sigma_{ \pm} \sigma_{ \pm} \sigma_{ \pm}: 2$ & & & & & & & & & & & & $\mathrm{X}$ \\
& & & & & & & & & & & &
\end{tabular}

The $X$ 's mark those elements of the relaxation superoperator that are non-zero. The number following the colon on the vertical axis indicates the number of elements that are grouped together by symmetry. The 
grouping is convenient because if each spin sees the same fluctuating field, then these elements will decohere at the same rate.

Control over the spin state is achieved through externally controllable Hamiltonians describing the interactions of the spin with either RF pulses or magnetic field gradients. The applied Hamiltonian has the following form, assuming only one nuclear species:

$$
H_{r f}=e^{i \omega_{\text {trans }} \sum_{i} \sigma_{z}^{i} / 2}\left(\frac{\omega_{1}}{2} \sum_{i} \sigma_{x}^{i}\right) e^{-i \omega_{\text {trans }} \sum_{i} \sigma_{z}^{i} / 2}
$$

where $w_{\text {trans }}$ is the carrier frequency of the RF pulse and $\omega_{1}$ is the effective amplitude of the RF field. The RF pulses can be applied non-selectively (to uniformly tilt all spins) by choosing $\omega_{1} \gg \Delta \omega$, or to selectively excite a single spin by choosing and modulating $\omega_{1}$ subject to $\omega_{1} \ll \Delta \omega$. Here $\Delta \omega$ is the difference between the transmitter frequency and that of the off-resonant spins. The selective version of $H_{r f}$ directly implements a useful version of the single qubit gate, provided that the pulse time is kept short compared to $1 / J$, where $J$ is the largest coupling constant.

It is useful to perform computation with gates easily implemented by the physical system. For example, many quantum algorithms are written in terms of Hadamard gates,

$$
U_{\text {Hadamard }}=\frac{1}{\sqrt{2}}\left(\begin{array}{cc}
1 & 1 \\
1 & -1
\end{array}\right)
$$

which in terms of a time independent Hamiltonian corresponds to a $180^{\circ}$ rotation about the $\vec{x}+\vec{z}$ direction. The Hadamard gate is used to rotate basis states into superpositions. Superpositions can also be reached through a $90^{\circ}$ rotation about the $x$-axis,

$$
U_{x, 90^{\circ}}=\frac{1}{\sqrt{2}}\left(\begin{array}{cc}
1 & -i \\
-i & 1
\end{array}\right)
$$

which is simpler to implement in NMR. Often a computation can be written in terms of gates that correspond more directly to the physical system.

A second example of physical gates are selective z-rotations which may be obtained from a series of RF pulses. This uses the fact that rotations around the $x$ and $y$ do not commute giving rise to z-rotations. Another possibility to implement z-rotation is to make a change of reference frame. Referring back to the block diagram of the spectrometer (and recalling that z-rotations commute with the internal Hamiltonian) we see that z-rotations can be accounted for in the reference phase of the individual transmitters and thus in general no explicit pulses are required. Keeping track of z-rotations becomes a book-keeping task.

A somewhat neglected part of QIP is the ability to introduce selective decoherence at will. This is convenient for preparing specific states and required for removing undesired information. Since NMR methods are ensemble measurements we may take advantage of the spatial distribution of the sample to create qubit selective decoherence. Decoherence is obtained by the action of a magnetic field gradient followed by molecular diffusion to remove all recoverable spatial correlation. The essential feature is that the spectrometer is normally equipped with a magnetic field gradient coil connected to a programmable current source, so the Hamiltonian is,

$$
H_{\text {grad }}=\frac{\partial B_{z}(t)}{\partial z} z \sum \frac{\gamma_{j}}{2} \sigma_{z}^{j}
$$

where $\gamma$ is the gyromagnetic ratio, and $z$ is the spatial location of the spin along the gradient direction. The action of the gradient is to create a linear phase ramp throughout the sample 109 which provides a decoherence mechanism with relaxation rate given by

$$
R=\gamma^{2}\left(\frac{d B_{z}}{d z}\right)^{2} \delta^{2}
$$

where $\delta$ is the time during which the spin system evolved in the magnetic field gradient, and the experiment consists of two strong, short and (effectively) opposite gradient pulses separated by the decoherence interval. 

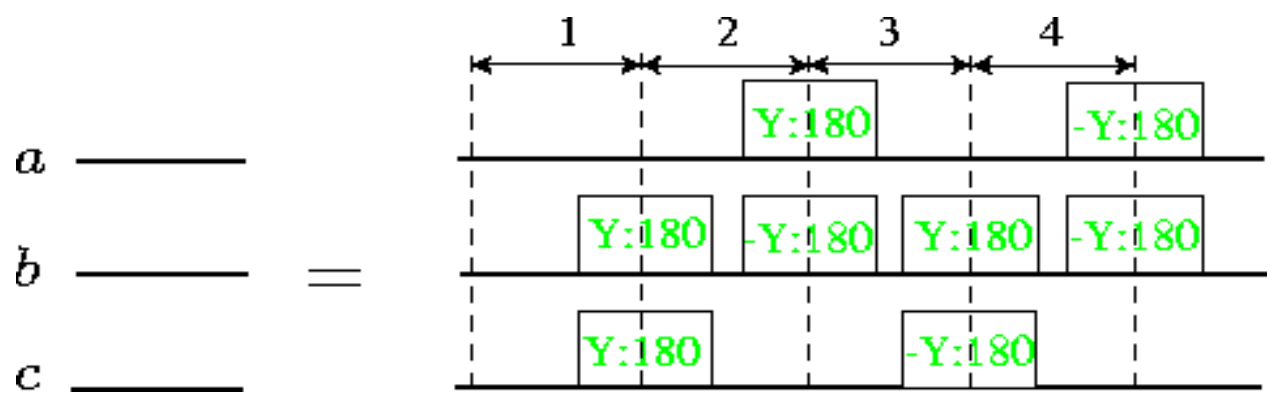

Figure 4: Implementation of a no-operation gate for three spins. The right-hand side shows the sequence of RF pulses which accomplishes this regardless of the Larmor frequencies and coupling constants. The pulses are a series of equally spaced parallel $180^{\circ}$ rotations about the $y$ axis. The net effect of the eight RF pulses is that except for the effects of decoherence, the average propagator is the identity. The left-hand side shows the equivalent computational gates as a staff with each qubit identified

\subsection{Unitary gates}

We will now show that through a combination of the internal Hamiltonian, modulated via time-dependent external Hamiltonians designed following the prescriptions of average Hamiltonian theory, any desired unitary operator can be achieved. It is only necessary to do this for a complete set of gates such as the C-not and single qubit gates (described above), but we will start by exploring a no-operation gate. A useful benchmark of QIP is the ability to suppress the internal Hamiltonian and thus to leave the system in whatever state it started in. This will also permit us to describe coherent averaging in some detail.

\subsubsection{No-operation}

One interesting feature of NMR compared to a device like the ion trap is that the coupling between qubits is always active. Refocusing methods have been invented to selectively turn off undesired couplings, so as to suppress their contribution to the effective propagator over some time interval. For example, starting with the internal Hamiltonian for a three qubit system:

$$
\begin{aligned}
& H_{3 \text { qubits }}=\frac{1}{2}\left(\omega_{a} \sigma_{z}{ }^{a}+\omega_{b} \sigma_{z}{ }^{b}+\omega_{c} \sigma_{z}{ }^{c}\right)+ \\
& \frac{\pi}{2} J_{a b} \sigma_{z}{ }^{a} \sigma_{z}{ }^{b}+\frac{\pi}{2} J_{a c} \sigma_{z}{ }^{a} \sigma_{z}{ }^{c}+\frac{\pi}{2} J_{b c} \sigma_{z}{ }^{b} \sigma_{z}{ }^{c}
\end{aligned}
$$

we can implement the identity operation using the sequence of RF pulses shown in Fig. $⿴$.

The effect of the sequence of Fig. 1 can be appreciated by breaking up the evolution into its four equal time parts, assuming that the RF pulses are effectively instantaneous, and transforming to a time-dependent interaction frame defined by the RF pulses (termed toggling frame Hamiltonian by Waugh 48]). The interaction-frame form of the internal Hamiltonian can be broken up into commuting parts. This can be visualized using the following table. The periods correspond to those shown in Fig.4 and the toggling frame states (with tilde) correspond to the interaction frame states under the actions of the RF pulses which are assumed to be delta function pulses.

\begin{tabular}{c|cccc|c} 
term & 1 & 2 & 3 & 4 & average \\
\hline$\tilde{\sigma_{z}}{ }^{a}$ & $+\sigma_{z}{ }^{a}$ & $+\sigma_{z}{ }^{a}$ & $-\sigma_{z}{ }^{a}$ & $-\sigma_{z}{ }^{a}$ & 0 \\
$\tilde{\sigma_{z}}{ }^{b}$ & $+\sigma_{z}{ }^{b}$ & $-\sigma_{z}{ }^{b}$ & $+\sigma_{z}{ }^{b}$ & $-\sigma_{z}{ }^{b}$ & 0 \\
${\tilde{\sigma_{z}}}^{c}$ & $+\sigma_{z}{ }^{c}$ & $-\sigma_{z}{ }^{c}$ & $-\sigma_{z}{ }^{c}$ & $+\sigma_{z}{ }^{b}$ & 0 \\
${\tilde{\sigma_{z}}}^{a}{\tilde{\sigma_{z}}}^{b}$ & $+\sigma_{z}{ }^{a} \sigma_{z}{ }^{b}$ & $-\sigma_{z}{ }^{a} \sigma_{z}{ }^{b}$ & $-\sigma_{z}{ }^{a} \sigma_{z}{ }^{b}$ & $+\sigma_{z}{ }^{a} \sigma_{z}{ }^{b}$ & 0 \\
${\tilde{\sigma_{z}}}^{a}{\tilde{\sigma_{z}}}^{c}$ & $+\sigma_{z}{ }^{a} \sigma_{z}{ }^{c}$ & $-\sigma_{z}{ }^{a} \sigma_{z}{ }^{c}$ & $+\sigma_{z}{ }^{a} \sigma_{z}{ }^{c}$ & $-\sigma_{z}{ }^{a} \sigma_{z}{ }^{c}$ & 0 \\
${\tilde{\sigma_{z}}}^{b}{\tilde{\sigma_{z}}}^{c}$ & $+\sigma_{z}{ }^{b} \sigma_{z}{ }^{c}$ & $+\sigma_{z}{ }^{b} \sigma_{z}{ }^{c}$ & $-\sigma_{z}{ }^{b} \sigma_{z}{ }^{c}$ & $-\sigma_{z}{ }^{b} \sigma_{z}{ }^{c}$ & 0
\end{tabular}

The terms of the Hamiltonian in the toggling frame Hamiltonian are shown for each of the four intervals. The average Hamiltonian is simply the time average of these states, since in this case the various toggling 

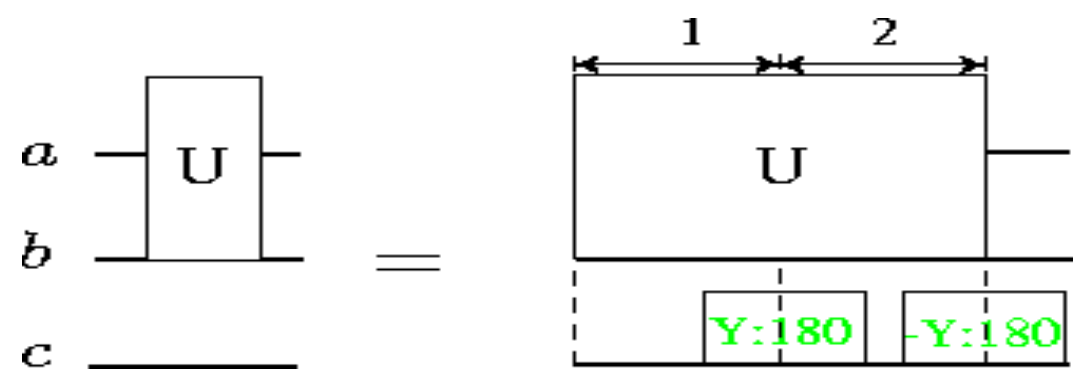

Figure 5: Sequence of RF pulses which accomplishes decoupling of spin $c$ from spins $a$ and $b$. This effectively removes the $c$ qubit (or spin) from the evolution. Qubit $c$ therefore stays in its original state while $a$ and $b$ evolve under their internal Hamiltonian.

frame Hamiltonians commute with each other over all periods. The action of the final pulses is not included in the table, but is important because in the absence of the internal Hamiltonian the effective propagator for the external Hamiltonian should be the identity operator.

Similar techniques can be used to selectively "turn off" interactions to a subset of the nuclei. One example of this can be used to simplify observation, by removing couplings during observation. This decoupling technique is widely used in NMR and can be thought of as an experimental method for "tracing out" unwanted degrees of freedom, as was done to obtain the spectra of Fig. 3. In Fig. [, a sequence of pulses demonstrate how this can be done. The following table gives the transformations of product operators of the 3 qubit Hamiltonian and their time average.

\begin{tabular}{c|cc|c} 
term & 1 & 2 & average \\
\hline$\tilde{\sigma_{z}}{ }^{a}$ & $+\sigma_{z}{ }^{a}$ & $+\sigma_{z}{ }^{a}$ & $\sigma_{z}{ }^{a}$ \\
$\tilde{\sigma_{z}}{ }^{b}$ & $+\sigma_{z}{ }^{b}$ & $+\sigma_{z}{ }^{b}$ & $\sigma_{z}{ }^{b}$ \\
$\tilde{\sigma_{z}}$ & $+\sigma_{z}{ }^{c}$ & $-\sigma_{z}{ }^{c}$ & 0 \\
${\tilde{\sigma_{z}}}^{a}{\tilde{\sigma_{z}}}^{b}$ & $+\sigma_{z}{ }^{a} \sigma_{z}{ }^{b}$ & $+\sigma_{z}{ }^{a} \sigma_{z}{ }^{b}$ & $\sigma_{z}{ }^{a} \sigma_{z}{ }^{b}$ \\
${\tilde{\sigma_{z}}}^{a}{\tilde{\sigma_{z}}}^{c}$ & $+\sigma_{z}{ }^{a} \sigma_{z}{ }^{c}$ & $-\sigma_{z}{ }^{a} \sigma_{z}{ }^{c}$ & 0 \\
$\tilde{\sigma_{z}}{ }^{b}{\tilde{\sigma_{z}}}^{c}$ & $+\sigma_{z}{ }^{b} \sigma_{z}{ }^{c}$ & $-\sigma_{z}{ }^{b} \sigma_{z}{ }^{c}$ & 0
\end{tabular}

The corresponding 3-qubit Hamiltonian is averaged to:

$$
H_{3 \text { qubits }}=\frac{1}{2}\left(\omega_{a} \sigma_{z}{ }^{a}+\omega_{b} \sigma_{z}{ }^{b}\right)+\frac{\pi}{2} J_{a b} \sigma_{z}{ }^{a} \sigma_{z}{ }^{b} .
$$

Thus if the evolution of the system is followed under the action of the average Hamiltonian one of the qubits appears to vanish from the system when observing the spectral lines associated with spins $a$ and $b$. For observation purposes it is sufficient to irradiate at $c$ 's resonance frequency with an RF field stronger than any of the scalar couplings involving $c$. Such decoupling techniques have inspired coherent approaches to other control problems such as dynamical decoupling or error correction for general noise [124, 123, 65.

\subsubsection{Control-not}

The C-not gate is a rotation of one qubit conditional on the state of a second. The desired propagator thus has the simple form

$$
\begin{aligned}
U_{\text {cnot }} & =\left(\begin{array}{cccc}
1 & 0 & 0 & 0 \\
0 & 1 & 0 & 0 \\
0 & 0 & 0 & 1 \\
0 & 0 & 1 & 0
\end{array}\right) \\
& =|0\rangle\langle 0|11+| 1\rangle\langle 1| \sigma_{x}
\end{aligned}
$$

This can be rewritten in terms of exponential operators (see general discussions by Somaroo et al. for more details 111, 95, 94])

$$
U_{\text {cnot }}=e^{-i \frac{\pi}{4}} e^{i \sigma_{x}{ }^{b} \frac{\pi}{4}} e^{i \sigma_{z}^{a} \frac{\pi}{4}} e^{-i \sigma_{z}^{a} \sigma_{x}{ }^{b} \frac{\pi}{4}}
$$



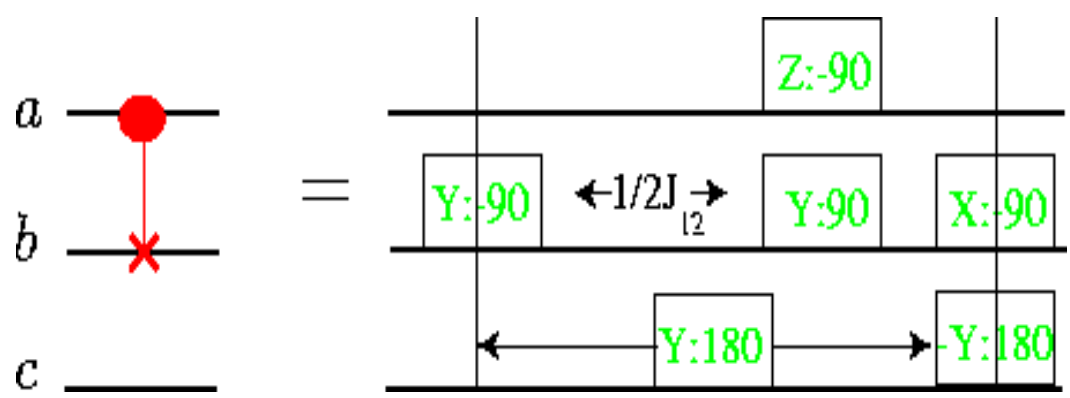

Figure 6: Translation of a C-not from qubit $a$ to qubit $b$ into a pulse sequence for nuclear spins. Note the required refocusing to suppress evolution involving spin $c$

all of which conveniently commute so that the order of implementation does not matter. The first term is an overall phase which we may neglect. The second term is a $90^{\circ} \mathrm{RF}$ pulse on the b-spin, and the third a phase shift of the a-spin. We have already seen how these can be applied. The last term is the one of interest, a two body interaction which induces a conditional rotation of the b-spin. The only two body Hamiltonian available in liquid state NMR is the scalar coupling $\sigma_{z}{ }^{a} \sigma_{z}{ }^{b}$. We may once again use AHT to transform this Hamiltonian into a propagator of the desired form, in this case by sandwiching a period of spin evolution under the scalar coupling with a pair of $90^{\circ}$ rotations of the b-spin about the y axis,

$$
e^{\left.-i \sigma_{z}^{a} \sigma_{x}^{(} b\right) \frac{\pi}{4}}=e^{-i \sigma_{y}{ }^{b} \frac{\pi}{4}} e^{-i \sigma_{z^{a}} \sigma_{z}{ }^{b} \frac{\pi}{4}} e^{i \sigma_{y}{ }^{b} \frac{\pi}{4}}
$$

Note that the internal Hamiltonian is always present, and while it is often neglected during non-selective pulses it must be included during selective pulses. In addition, the chemical shifts of other spins and couplings other than between spins $a$ and $b$ should be refocused during the coupling evolution period. This is normally accomplished via a series of $180^{\circ}$ rotations similar to those used during the no-operation gate. A pulse sequence which accomplishes this for three spins is shown in Fig. 6 .

\subsection{Measurement}

NMR measurements are weak (as opposed to strong projective measurements), therefore we can follow the evolution of the spin magnetization over time with only small distortions being introduced by the detection coil 16]. Because the coil is classical we may treat the coupling between it and the spin system as an example of magnetic induction. The measured electro-motive force (emf) is then,

$$
e m f=-\frac{d}{d t} \int \frac{B_{1} \cdot M(t)}{\left|B_{1}\right|} d \Omega,
$$

where $\frac{B_{1}}{\left|B_{1}\right|}$ is the normalized intensity of the RF field generated by the detection coil, $M(t)$ is the macroscopic local spin magnetization, and the integral is over the entire volume. Since the RF coil generates a dipolar field, only those portions of density matrix that are dipolar and oriented along the field $B_{1}$ will couple. The signal is measured over time by currents induced by the rotating bulk magnetization in a transverse coil tuned to the resonance frequency, so that the measured signal can be given as a trace

$$
\operatorname{obs}(t)=\operatorname{Tr}\left[\sum_{j} \sigma_{+}^{j} \rho\right],
$$

where $\sigma_{+}{ }^{j}=\sigma_{x}{ }^{j}+i \sigma_{y}{ }^{j}$. The density matrix $\rho$ is modulated in time by the internal Hamiltonian, and is implicitly shifted to the rotating frame associated with the resonance frequency. As a result, in product operator terms, the following six operators

$$
\begin{array}{ll}
\sigma_{x}^{a} 111 & , \quad \sigma_{y}^{a} 111 \\
11 \sigma_{x}^{b} 11 & , \quad 1 \sigma_{y}{ }^{b} 1
\end{array}
$$




$$
111 \sigma_{x}^{c} \quad, \quad 111 \sigma_{y}^{c}
$$

are directly observable for the three spin system.

Other product operators are indirectly observable since the NMR signal is detected over time, and with normally evolve under the influence of the internal Hamiltonian. Thus the directly observed product operators for spin a shows a modulation from coupled spins via the action of the scalar coupling. For example,

$$
\sigma_{x}{ }^{a} \sigma_{z}{ }^{b} 11 \stackrel{\frac{\pi}{2} J_{a b \sigma_{z}}{ }^{a} \sigma_{z}{ }^{b}}{\longrightarrow} \cos \left[\frac{\pi}{2} J_{a b} t\right] \sigma_{x}{ }^{a} \sigma_{z}{ }^{b} 11+\sin \left[\frac{\pi}{2} J_{a b} t\right] \sigma_{y}{ }^{a} 111
$$

As a result, an additional eighteen terms which evolve in and out of directly observable terms are indirectly observable. This includes $\sigma_{x}{ }^{a} \sigma_{z}{ }^{b}$, but not, for example, $\sigma_{x}{ }^{a} \sigma_{x}{ }^{b}$ which does not evolve under the scalar coupling Hamiltonian.

The decoupling methods from Sec. 3.3.1 suppress the scalar coupling to the decoupled spin and thus, so-called anti-phase states such as $\sigma_{x}{ }^{a} \sigma_{z}{ }^{c}$ will not be observed in the measurement. This is consistent with considering decoupling as a tracing operation.

For deterministic algorithms, it is sufficient to measure a single spin. For small Hilbert spaces, however, it is often convenient to measure the full density matrix when exploring the performances of a QP. For most QPs where the measurement involves collapsing into a measurement basis, reading out the density matrix involves a tomographic procedure of repeated measurements as a function of a rotation of the system into the measurement basis. The density matrix is then reconstructed by inverting the measurement results. In NMR, since the measurement is weak, all transverse dipolar degrees of freedom can be read out directly and in one step. The result is that state tomography can be achieved in fewer steps and the density matrix reconstructed via spectral fitting. The method involves appending readout pulses to the end of the calculation to rotate non-observable operators into observable ones. For the three qubit system under discussion, seven measurements (with different readout pulses) are sufficient to completely characterize the density matrix. Following the measurement of a complete set of spectra, these may be fitted to extract the complete description of the density matrix.

\subsection{Non-unitary operations}

A desirable operation for a $\mathrm{QP}$ is a strong projective measurement. In cases where the projective measurement does not contribute toward the final answer, such as is the case in error correction procedures, only its effect on the state, not its outcome, is required. This effect can be mimicked by decohering the off-diagonal elements in a measurement basis. This can be accomplished through natural decoherence after having rotated the spins in the desired basis [89], or by the action of a magnetic field gradient followed by molecular diffusion to remove all recoverable spatial correlations. Thus ensemble dynamics makes it is

possible to mimic strong measurement processes, although unfortunately this cannot be used as a means of repolarizing the system since a mixed state results.

\subsection{Pseudo-pure states}

One example where controlled decoherence is necessary is the preparation of pseudo-pure states. At room temperature the NMR spin system is in a highly mixed state since $\Delta E / k T$ is of order $10^{-5}$. The sample consists of about $10^{18}$ molecules and the state must be described by a density matrix which at equilibrium for a single nuclear species is

$$
\rho_{e q}=\frac{1}{Z} e^{-H / k T} \approx \frac{1}{Z} \mathbb{1}+\epsilon_{1} \sum_{i} \sigma_{z}^{i}
$$

where $Z$ is a normalization factor and recall that $\omega / J \approx 10^{6}$ so to a good approximation the bilinear term in the Hamiltonian does not influence the populations. As long as relaxation is unimportant the identity portion of the density matrix is a constant, and is unobservable. In a homogeneous magnetic field, we have 


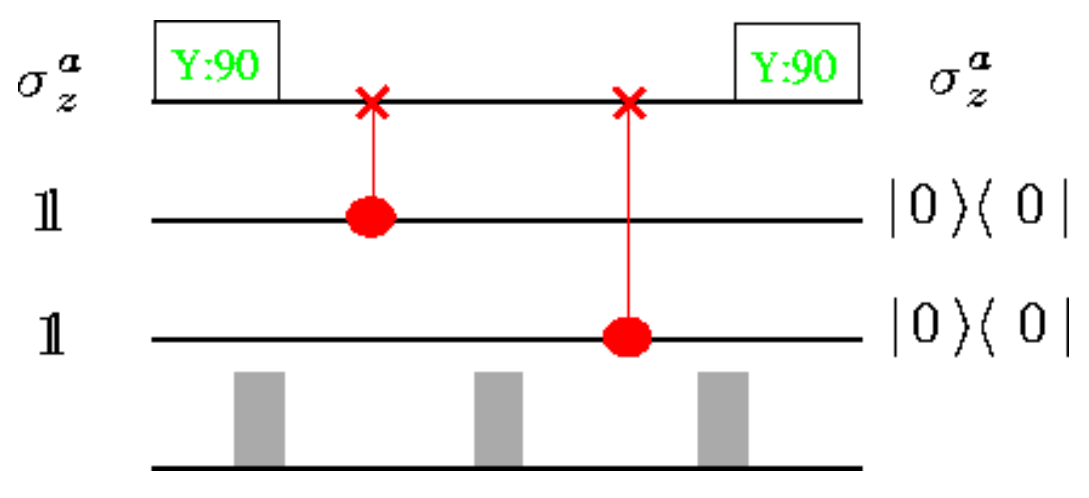

Figure 7: Pulse sequence to create a pseudo-pure state from the state $\sigma_{z}{ }^{a} 111$. The grey filled rectangle in the bottom line correspond to gradient pulses. The relative area of the three gradient pulses are shown in the figure.

no useful control over the identity part of the equilibrium density matrix, so NMR methods rely on pseudopure states whose dynamics are equivalent to those of pure states with respect to weak measurements. An example of a pseudo-pure state is given by

$$
\rho_{p p}=\frac{1}{Z} \mathbb{1}+\epsilon_{2} \prod_{i}|0\rangle_{i i}\langle 0|,
$$

which has the property that its traceless part is up to a scalar constant equal to that of a pure state. The scalar constant is the intensity of the pseudo-pure state. Methods for creating pseudo-pure states on all spins have intensity decreasing exponentially with the number of qubits. This is why for general quantum computing, liquid state NMR is limited to about ten spins.

Instead of generating the pseudo-pure state of Eq. 31, it is usually simpler to create

$$
\rho_{x p p}=\lambda \mathbb{1}+\epsilon_{3} \sigma_{x}{ }^{0} \prod_{i \geq 1}|0\rangle_{i i}\langle 0|
$$

This state can either be used as a standard pseudo-pure state on one less spin (the measurement needs to be somewhat modified), or in many cases it can serve directly as one of the inputs in benchmarking an algorithm involving coherent processing of one qubit [64]. Decoherence operations such as those available with gradients yield particularly simple means for creating states of this form. A procedure which accomplishes this for three spins is given in Fig. 7.

We can again use AHT to follow the gradient Hamiltonian in the toggling frame of the C-not pulses for the sequence of Fig. . There are three intervals, and the evolution is by a position dependent rotation about the $\mathrm{z}$ axis.

\begin{tabular}{c|ccc|c|c} 
& 1 & 2 & 3 & average & $2 \theta_{1}=\theta_{2}=2 \theta_{3}=\theta$ \\
\hline$E_{+}^{a} E_{+}^{b} E_{+}^{c}$ & $\theta_{1}$ & $-\theta_{2}$ & $\theta_{3}$ & $\theta_{1}-\theta_{2}+\theta_{3}$ & 0 \\
$E_{+}^{a} E_{+}^{b} E_{-}^{c}$ & $\theta_{1}$ & $-\theta_{2}$ & $-\theta_{3}$ & $\theta_{1}-\theta_{2}-\theta_{3}$ & $-\theta$ \\
$E_{+}^{a} E_{-}^{b} E_{+}^{c}$ & $\theta_{1}$ & $\theta_{2}$ & $-\theta_{3}$ & $\theta_{1}+\theta_{2}-\theta_{3}$ & $\theta$ \\
$E_{-}^{a} E_{+}^{b} E_{+}^{c}$ & $\theta_{1}$ & $-\theta_{2}$ & $\theta_{3}$ & $\theta_{1}-\theta_{2}+\theta_{3}$ & 0 \\
$E_{+}^{a} E_{-}^{b} E_{-}^{c}$ & $\theta_{1}$ & $\theta_{2}$ & $\theta_{3}$ & $\theta_{1}+\theta_{2}+\theta_{3}$ & $2 \theta$ \\
$E_{-}^{a} E_{+}^{b} E_{-}^{c}$ & $\theta_{1}$ & $-\theta_{2}$ & $-\theta_{3}$ & $\theta_{1}-\theta_{2}-\theta_{3}$ & $-\theta$ \\
$E_{-}^{a} E_{-}^{b} E_{+}^{c}$ & $\theta_{1}$ & $\theta_{2}$ & $-\theta_{3}$ & $\theta_{1}+\theta_{2}-\theta_{3}$ & $\theta$ \\
$E_{-}^{a} E_{-}^{b} E_{-}^{c}$ & $\theta_{1}$ & $\theta_{2}$ & $\theta_{3}$ & $\theta_{1}+\theta_{2}+\theta_{3}$ & $2 \theta$
\end{tabular}

where $E_{ \pm}^{i}=\left(\mathbb{1} \pm \sigma_{z}^{i}\right) / 2$. Putting this all together and writing the gradient Hamiltonian out explicitly, the averaged gradient Hamiltonian introduced by the above sequence is,

$$
\bar{H}_{\text {grad }}=\gamma \frac{\partial B_{z}}{\partial z} z \sigma_{z}{ }^{a}\left(-E_{+}{ }^{b} E_{-}{ }^{c}+E_{-}{ }^{b} E_{+}{ }^{c}+2 E_{-}{ }^{b} E_{-}{ }^{c}\right)
$$


The effect of the dependence on $z$ and subsequent diffusion is that the input state projects to the desired pseudo-pure state.

There are many other approaches to create pseudo-pure states, including temporal averaging schemes, and logically labeling the state dependent on another spin 117, 118, 61, 41, 64. The polarization of all of these falls off exponentially with the number of qubits.

\subsection{Achievements of liquid state NMR QIP}

One reason why NMR QIP has had such a head start over other approaches to QIP is that NMR spectroscopy has been used for coherent manipulations for decades. Indeed much work on NMR is directed towards coherently controlling quantum states so as to extract specific chemical information from complex systems. This has led the NMR community to develop powerful analytic tools for understanding spin dynamics.

In the weak coupling limit, where qubits are most easily implemented in terms of nuclear spins, the product operator formalism 114, 122, 90] provides a complete description of the dynamics. Not only is this extremely useful experimentally, but it also exactly describes the desired dynamics of a quantum computer. Of course for NMR spectroscopy the focus is on single-spin operations and scalar couplings, and for QIP it is on single qubit operations and C-not gates. In the case of more complex spin systems, either with non-negligible strong coupling or with spins $>\frac{1}{2}$, the dynamics is often expanded in a fictitious spin $\frac{1}{2}$ formalism [1], which essentially is a mapping of the physical system into qubits in the manner suggested for quantum simulation outlined in Fig. 1. Beyond a certain complexity it is far too difficult to map the dynamics into distinguishable qubits and the focus turns to thermodynamic properties of the spin system via the concepts of spin temperature 44. A major challenge of QIP is to use these larger systems for information processing.

We have seen repeated examples of how average Hamiltonian theory is related to quantum simulation and extremely useful for choosing specific implementations of quantum gates. Although limited space has not permitted a detailed discussion, similar control may be gained over non-unitary superoperators through the use of average Liouvillian theory [42, 120]. In general relaxation is understood through superoperator theories [51], and these ideas are just now being combined with QIP in the context of noiseless subspaces 134, 71] and subsystems [65. Interestingly the concepts of quantum error correction 106] do not seem to have been anticipated in even a primitive sense by the NMR community. This is perhaps an example of where complex ideas can only be reached through a formal information theory approach.

The same features that make QIP possible, namely the ability to strongly modulate the spin dynamics on a time scale short compared to decoherence, has lead to a wealth of NMR methods aimed at specific chemical and physical problems. Many of the experimental techniques that have made the NMR implementations of QIP proceed so quickly are well established in NMR. There are very robust methods for creating selective pulses $126,98,39,83$, and these have reached a very high precision for the purposes of magnetic resonance imaging. Almost every NMR experiment today makes use of some form of coherent control: (1) to transfer polarization for sensitivity enhancement [82, 34], (2) to transfer coherence for correlation experiments [11, 8], (3) to selectively suppress coherence for solvent signal elimination and to limit the dynamic range of the signal [17, and (4) to select multiple quantum pathways to observe specific desired subsystems of spins 18, 127. Such control is also used to tailor the dynamics: (5) for decoupling undesired spins [70, 128, (6) for recoupling spins whose chemical shift differences are greater than the coupling between them [19] and (7) to selectively remove dipolar [129] or even quadrupolar [84] interactions. Most of these methods have found applications in QIP, and the precision of control required by QIP implies that we will have to make use of all possible means of coherent control.

NMR spectroscopy has not exclusively focused on chemical and medical applications, it has also been used as a probe of quantum dynamics. Many experiments have been carried out to explore the structure of quantum dynamics. The field has struggled with the question of bounds on the efficiency of transformations such as polarization transfer [112] and on finding means to achieve these experimentally [113]. QIP-like explorations include interferometric measurement of spinor dynamics 117 (the creation of logically labeled pseudo-pure states were needed for these) and explorations of geometric phase 118]. There is also a recent NMR implementation of geometric phase as a controlled gate [55].

There has also been a series of explorations of quantum complexity for systems of dipolarly coupled spins conducted in both liquid crystals [102] and the solid state 85. It is the realization that NMR studies of 
dipolarly coupled spins provides a test bed for complex quantum dynamics that has led Chuang [133] and us to propose next generation QIP devices built from liquid crystals and solids, respectively. The solid state also offers an ideal test bed for larger studies of multi-body dynamics and of the transition from quantum to classical behavior 136.

In this contribution we cannot do justice to all the NMR QIP experiments that have been realized and certainly not to 50 years of NMR spectroscopy. Clearly without the accumulated knowledge of NMR spectroscopy theory, methods and instrumentation, the field of NMR QIP would not be so advanced. Fortunately there are excellent and detailed descriptions of NMR [1, 103, 46, 37, 36] The following table summarizes the NMR QIP experiments published to date.

\begin{tabular}{|c|c|c|c|}
\hline \# of qubits & Algorithms & Year & Reference \\
\hline \multirow[t]{8}{*}{2} & Gates & 1996 & (25, 21, 24, 23, 95, ,79, 55 \\
\hline & Database Search & 1998 & [54, 20, 133] \\
\hline & Deutsch-Josza & 1998 & 53,22 \\
\hline & Quantum Simulation & 1999 & "110 \\
\hline & Quantum Detecting Code & 1999 & 69 \\
\hline & Quantum Fourier Transform & 1998 & 1130, 40 \\
\hline & Dense Coding & 1998 & 38 \\
\hline & Quantum Detecting Code & 1999 & 69 \\
\hline \multirow[t]{7}{*}{3} & GHZ state & 1997 & 67,88 \\
\hline & Quantum Error Correction & 1997 & [26] \\
\hline & Quantum Teleportation & 1997 & 89 \\
\hline & Deutsch-Josza & 1998 & 35,72 \\
\hline & Quantum Simulation & 1999 & 120 \\
\hline & Quantum Fourier Transform & 1998 & $\mid$ \\
\hline & Quantum Eraser & 1998 & 119 \\
\hline 4 & $\mathrm{C}^{3}$-not Gate & $\overline{1999}$ & 95 \\
\hline 5 & Deutsch-Josza & 1999 & 80 \\
\hline 6 & Decoupling & 1998 & 77 \\
\hline 7 & Benchmark & 1999 & 64 \\
\hline
\end{tabular}

\section{Limitations of liquid state NMR QIP}

Although liquid state NMR is extremely useful to demonstrate coherent control and bring theoretical quantum computation to the laboratory it has the following limitations:

1) The non-scalability of pseudo-pure state preparation. As the number of qubits increases, the signal resulting from the pseudo-pure state preparation decreases exponentially. This decay quickly becomes prohibitive and renders quantum error correction ineffective since it requires ancillas in pseudo-pure states.

2) The large ratio of the gate time over the decoherence time (of order .01). This is sufficient for proofof-principle experiments but too small for computationally interesting algorithms.

3) The difficulty of resetting qubits. In order to iterate quantum error correction, "fresh" qubits must be supplied. This can be achieved by either having initially a large supply that remains perfectly polarized or by refreshing the qubits by setting them back to a fiducial state. Liquid state NMR does not seem to provide a mechanism for resetting qubits at will.

4) As we enlarge the number of qubits the spread of Larmor frequencies (limited by the chemistry) will have to increase accordingly in order to preserve the same degree of control. Although this can be circumvented, doing so is difficult 74]. Unfortunately nature limits the range of chemical shifts to about $200 \mathrm{ppm}$ of the resonance frequency for carbon, sufficient to distinguish only about 20 carbon nuclei for QIP purposes at reasonable field strengths and operation times. 
The first generation of QPs based on liquid state NMR has been very useful as a first step in a program towards a quantum computer. But faced with the above limitations we now propose to move to a solid state NMR-based device which we believe will carry QIP beyond 10 qubits.

\section{Introduction to solid state NMR}

Before presenting the next generation of NMR quantum information processors we quickly survey the essential elements distinguishing liquid and solid state NMR. The solid state offers four main advantages for QIP. First, the system can be highly polarized increasing the sensitivity necessary to read out the results of computations involving many qubits. Second, the decoherence rates can be made slower. Third, couplings between spins are higher, thereby permitting both faster and more accurate operations, so that algorithms of much greater complexity can be implemented. Finally, there are mechanisms which can be used for dynamically resetting qubits, thus making it possible to remove information from a system and to implement error-correction efficiently.

From a spectroscopy point of view the main differences between liquid and solid state NMR are:

1) The dipolar couplings are resolved.

2) Spin diffusion contributes significantly to the dynamics.

3) The chemical shift needs to be described by a tensor.

4) The spin lattice relaxation times are typically very long.

5) The transverse (phase) decoherence time is dominated by spin-spin interactions which can be refocused by special purpose RF pulse sequences.

6) Methods are known for creating very high non-thermal polarizations.

In the liquid state the interaction between distinguishable nuclear spins is mediated through electrons as an indirect interaction or J-coupling. In the solid state, the direct dipole-dipole coupling is time independent and thus observed. The internal Hamiltonian in the solid state has the form,

$$
\begin{aligned}
H_{\text {solid }}=\sum_{i} & \frac{\omega_{i}(\theta, \phi)}{2} \sigma_{z}{ }^{i}+\sum_{j} \sum_{k} \frac{\gamma_{j} \gamma_{k}}{4 r_{j, k}^{3}}\left(3 \cos ^{2} \theta_{j, k}-1\right) \sigma_{z}^{j} \sigma_{z}^{k} \\
& -\sum_{j} \sum_{k} \frac{\gamma_{j} \gamma_{k}}{16 r_{j, k}^{3}}\left(3 \cos ^{2} \theta_{j, k}-1\right)\left(\sigma_{+}^{j} \sigma_{-}^{k}+\sigma_{-}^{j} \sigma_{+}^{k}\right)
\end{aligned}
$$

where $\theta$ and $\phi$ are the Euler angles associated with the orientation of the molecule in the external magnetic field and $\theta_{j, k}$ and $r_{j, k}$ are the angle and magnitude of the difference vector between nuclei $j$ and $k$ with respect to the external field.

In the homonuclear case the "flip-flop" $\left(\sigma_{+} \sigma_{-}+\sigma_{-} \sigma_{+}\right)$term is important and results in spin diffusion, while in the heteronuclear case this term is non-secular and may be dropped from the Hamiltonian. The heteronuclear case is thus very similar to the liquid state situation described above, except that both the dipolar couplings and chemical shifts have an orientation dependence (relative to the external magnetic field). It will therefore be important to use single crystals so that there is a unique mapping of the spin system to the resonance frequency, or, alternatively to apply magic angle sample spinning [7 to remove the orientation dependence. If a single crystal is used, then the sample must crystallize with only one molecule per unit cell.

In the homonuclear case the dipole-dipole coupling leads to a mixing of states that often prevents the chemical shift term from being useful for spectrally separating chemically distinct spins. In addition, the flip-flop term leads to an apparent short transverse decoherence rate. The flip-flop dynamics is however perfectly coherent and should be viewed in the light of recording the history of spin evolution. However, it has the reputation of being decoherent because it is normally studied in samples where the homonuclear chemical shift differences are not resolved and thus the observed Hilbert space is that resulting from a trace over what are in fact distinct spins. Via this trace operation the coherent flip-flop dynamics are artificially 


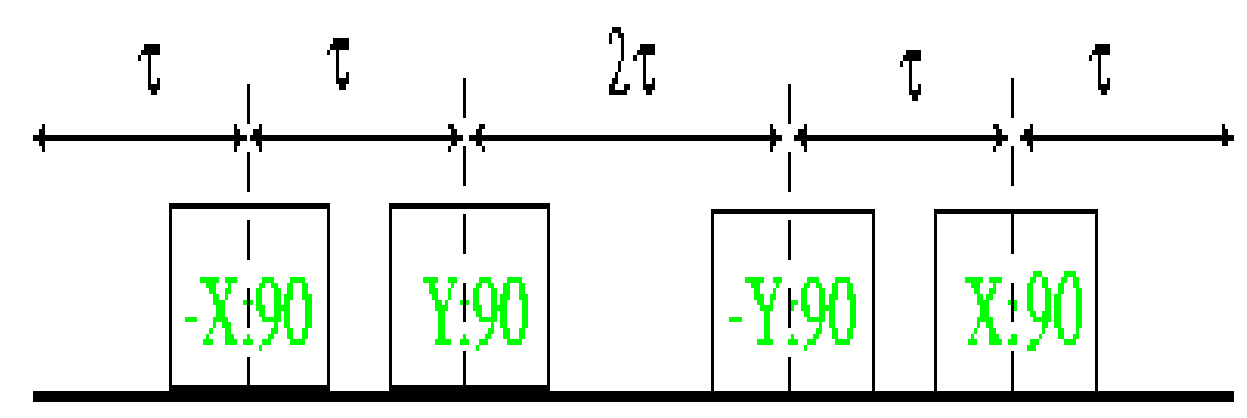

Figure 8: WAHUHA pulse sequence to reduce the dipolar interaction between spins. The sequence is applied to all nuclei.

mapped into an apparently non-unitary evolution. For most samples the true transverse decoherence rates are extremely challenging to measure requiring suppression of the dipolar interaction over the duration of the measurement [27].

To control and observe the spin dynamics in the presence of dipolar coupling one needs to employ multiple-pulse coherent averaging which independently rescales the dipolar and chemical shift terms of the Hamiltonian in the AHT. A simple and descriptive example is the WAHUHA sequence [129], the effects on the terms of the Hamiltonian in the toggling frame are,

\begin{tabular}{l|ccccc|l} 
term & 1 & 2 & 3 & 4 & 5 & average \\
\hline$\sigma_{z}{ }^{a}$ & $\sigma_{z}{ }^{a}$ & $\sigma_{y}{ }^{a}$ & $\sigma_{x}{ }^{a}$ & $\sigma_{y}{ }^{a}$ & $\sigma_{z}{ }^{a}$ & $\frac{1}{3}\left(\sigma_{x}{ }^{a}+\sigma_{y}{ }^{a}+\sigma_{z}{ }^{a}\right)$ \\
$\sigma_{z}{ }^{b}$ & $\sigma_{z}{ }^{b}$ & $\sigma_{y}{ }^{b}$ & $\sigma_{x}{ }^{b}$ & $\sigma_{y}{ }^{b}$ & $\sigma_{z}{ }^{b}$ & $\frac{1}{3}\left(\sigma_{x}^{b}+\sigma_{y}{ }^{b}+\sigma_{z}{ }^{b}\right)$ \\
$\sigma_{z}{ }^{a} \sigma_{z}{ }^{b}$ & $\sigma_{z}{ }^{a} \sigma_{z}{ }^{b}$ & $\sigma_{y}{ }^{a} \sigma_{y}{ }^{b}$ & $\sigma_{x}{ }^{a} \sigma_{x}{ }^{b}$ & $\sigma_{y}{ }^{a} \sigma_{y}{ }^{b}$ & $\sigma_{z}{ }^{a} \sigma_{z}{ }^{b}$ & $\frac{1}{3} \sigma^{a} \cdot \sigma^{b}$ \\
$\sigma^{a} \cdot \sigma^{b}$ & $\sigma^{a} \cdot \sigma^{b}$ & $\sigma^{a} \cdot \sigma^{b}$ & $\sigma^{a} \cdot \sigma^{b}$ & $\sigma^{a} \cdot \sigma^{b}$ & $\sigma^{a} \cdot \sigma^{b}$ & $\sigma^{a} \cdot \sigma^{b}$
\end{tabular}

From the above scheme it can be seen that the homonuclear dipolar coupling averages to

$$
\propto \sigma^{a} \cdot \sigma^{b}-3\left\langle\sigma_{z}^{a} \sigma_{z}{ }^{b}\right\rangle=0,
$$

though the chemical shift term remains. The averaged chemical shift Hamiltonian is now oriented along the $\vec{x}+\vec{y}+\vec{z}$ direction. In practice more complex sequences are used that include finite pulse width effects and also higher order terms in the Magnus expansion.

Related sequences such as the magic echo [101] rely on the convenient property that in a strong spin locking field the dipolar Hamiltonian is scaled by a factor -0.5. Thus under spin locking conditions the dipolar Hamiltonian can be refocused, because the coherent part of the dynamics appears to run backwards.

The spin-lattice relaxation times of diamagnetic crystalline solids below their Debye temperature are generally very long (of the order of hours) and are frequently dominated by the presence of defects [15]. A common defect is a color center containing free electrons. Since the electron spin correlation time is short, this acts as a locally fluctuating field that decoheres nearby nuclear spins. This local spin magnetization gradient is then carried through the lattice by the flip-flop term of the homonuclear dipolar coupling. Indeed it has been observed that in the presence of off-resonance excitation (to quench spin diffusion) the $T_{1}$ becomes longer, and this can be attributed to the fact that relaxation is mediated by magnetization transport and without spin diffusion there is no way for the nuclear magnetization to reach the electron relaxation sinks.

Stable free electrons like those in color centers are also valuable for providing a source of polarization. The electron magnetic moment is about 1800 times that of the proton, so at $4^{\circ} \mathrm{K}$ the electron spins are highly polarized. This polarization can be transferred coherently or via anisotropy in decoherence to the 
local nuclear spins, from which spin diffusion can carry the polarization to the more distant portions of the sample. The electron relaxation times are short, and so the effective heat capacity of the electron spin bath is high enough to polarize many nuclear spins.

\section{Next generation QIP based on solid state NMR}

\subsection{General scheme}

To build up to a prototype QP capable of non-trivial quantum algorithms, simulations, and decoherence studies, we propose a next generation of NMR QPs based on solid state. In this section we first introduce the general scheme, then give a detailed description of the key steps and components. A large scale QP can be envisioned on the basis of the following device consisting of an ensemble of microscopic, identical QPs. The heart of the system is an n-qubit QP composed of a set of spin $\frac{1}{2}$ nuclei in a precisely engineered arrangement associated with the chemistry of a designed molecule. Individual QPs are held and aligned in a lattice in which the only active nuclear spins are deuterium nuclei. To achieve uniformity of the lattice, it is convenient to use an isotopic variant of the molecule that is used for the QP. If the spins used for QIP are given by ${ }^{1} \mathrm{H}$ and ${ }^{13} \mathrm{C}$, a useful variant is fully deuterated and unlabeled. The entire ensemble is thus a single crystal of the deuterated molecules with a very dilute concentration of suitably labeled versions. The concentration of QPs is kept low enough that the individual QPs do not communicate with one another. Spectroscopic studies of such molecular preparations are well known, and are useful when the goal is to obtain well resolved proton spectra from complex molecules [108]. Since the individual QPs are in close proximity to the deuterated bath, an important component of this approach is to completely decouple the deuterons from the spin $\frac{1}{2}$ nuclei via multiple-pulse sequences. Methods for such decoupling sequences are well known 92. An illustration of our proposal is in Fig. 9.

The molecules that make up the individual QPs are designed so that the dipolar couplings between spins remains strong (of the order of $\mathrm{kHz}$ ) while the spectral separation between nuclei is sufficiently large for the qubits to remain individually addressable. This is facilitated by the fact that in the solid state both the chemical shift and dipolar coupling retain their anisotropy and hence reorientation of the sample relative to the external magnetic field provides a convenient way for controlling their relative strengths. An important goal is to ensure that the spectral separation between any two qubits is appreciably larger than their dipolar coupling. This should be achievable in principle, due to the fact that the dipolar coupling goes to zero when the inter-nuclear vector is at the magic angle to the applied magnetic field.

An additional simplification occurs at high polarizations in that the flip-flop term of the Hamiltonian is quenched and thus configurational broadening of the resonance line is avoided both from within the QC and with the deuterated lattice (external to the QC) which, as stated, is actively decoupled.

The deuterated lattice can include color centers (for example, stable free electrons can be introduced through gamma irradiation). They are used to generate large non-thermal polarizations in the nuclear spins through the well established method of dynamic nuclear polarization (DNP) 132. The spin $\frac{1}{2}$ nuclei that make up the QP are polarized in a two step process. First, the deuterium lattice is polarized by transfer of the free electron polarization. Then this polarization is transferred to the QP's spins via cross polarization [50]. Since the heat capacity of the lattice is much greater than that of the dilute molecules of the QP this transfer will polarize the QP close to the initial polarization of the electrons.

To increase the DNP signal enhancement the system is kept at $-8^{\circ} \mathrm{K}$, at which temperatures the electron spins are nearly in a pure state. The system is also arranged so that the QPs are far enough apart that they do not directly see the color centers, which under normal circumstances would be the primary source of decoherence (the crystal is well below its Debye temperature). At such low temperatures the spin-spin interactions are more important than the phonon motions and the only fluctuating magnetic fields arise from the lifetimes of the z-component of the electron spin, which has a relatively short spin-lattice relaxation time. The double quantum decoupling method mentioned earlier also decouples the deuterium spins from one another so that spin transport through the lattice is quenched and the color centers no longer play a significant relaxation role 81]. Thus the decoherence times of the QPs will be very long (of order $10^{3}$ seconds). Notice that the electrons play no role in the computation and their short decoherence time is in fact a benefit for rapid state preparation without in any way limiting the achievable computational complexity. 


\section{Malonic acid}
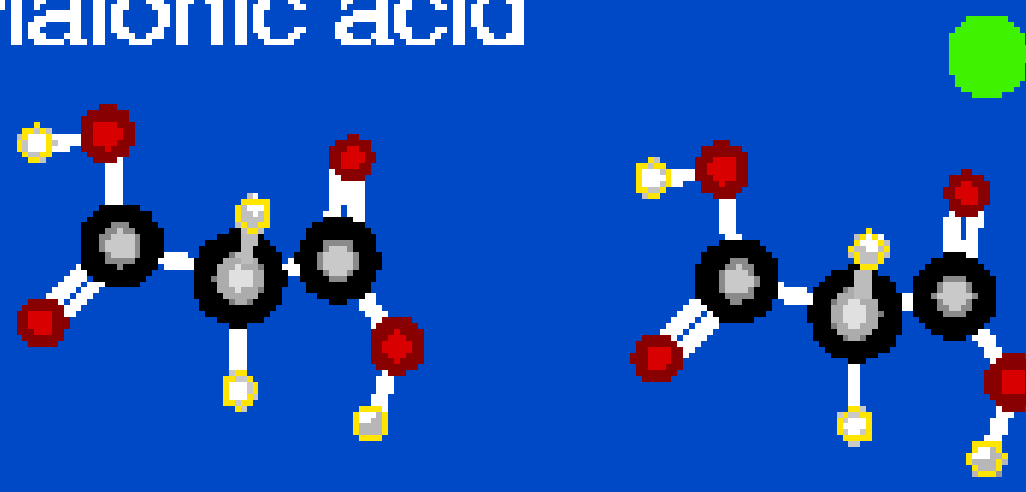

\section{(Color Cente}
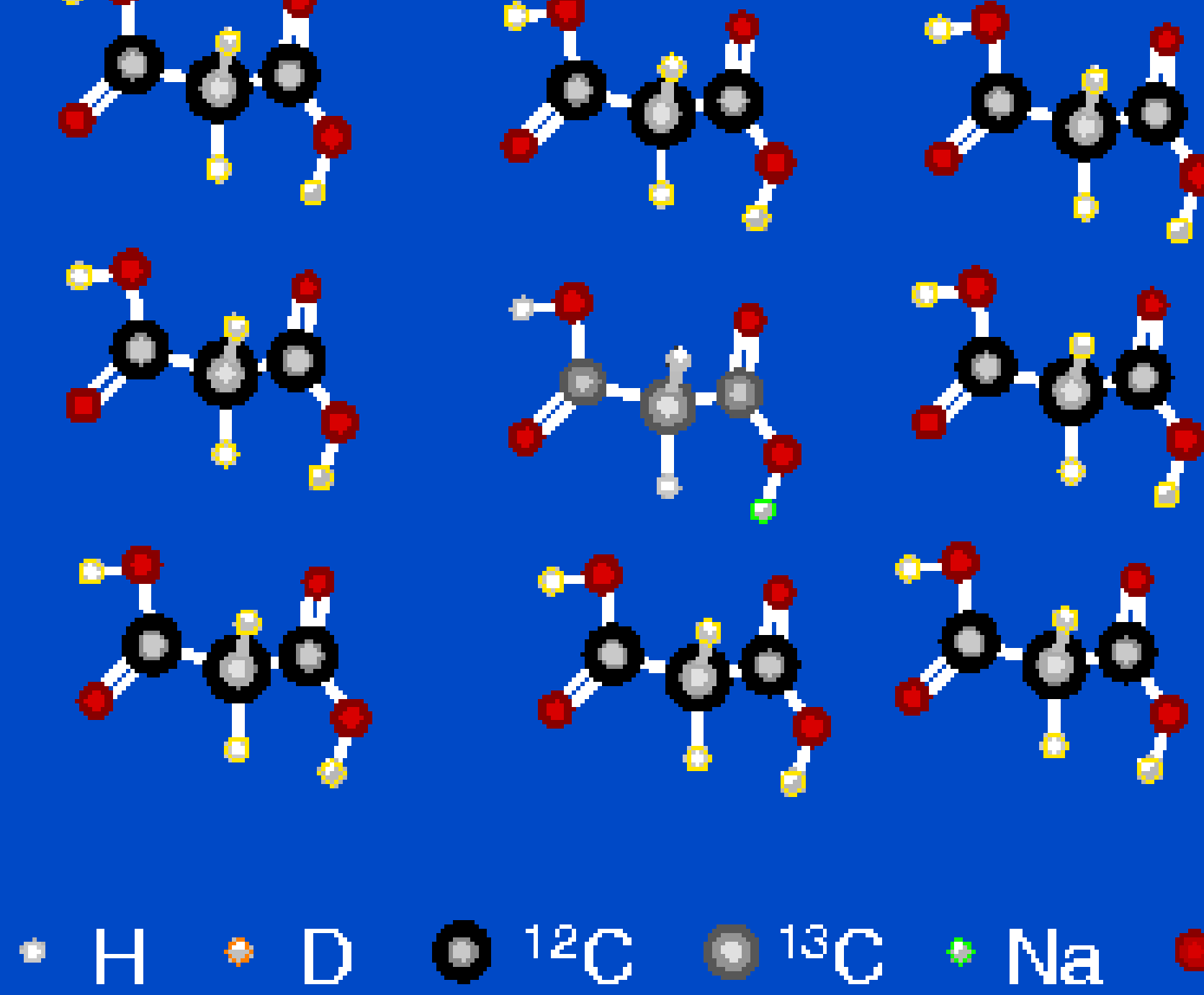

Figure 9: Solid state NMR quantum processor using malonic acid for illustration. An idealized lattice of the molecules is shown. One of the molecules has ${ }^{1} \mathrm{H},{ }^{13} \mathrm{C}$ and one sodium atom, whose nucleus serves as a resettable qubit. This molecule is immersed in a background lattice of deuterated and ${ }^{13} \mathrm{C}$ depleted crystal of the same acid with no sodium. Color centers are included to generate large non-thermal polarizations. 
In future implementations it may be feasible to include an erasable quantum register. This permits the complete purification of states through polarization pumping [104], the efficient implementation of quantum error-correction codes, and provides a source of decoherence to mimic the effect of strong measurements. This register could be composed from a set of quadrupolar spins in the molecule of a QP that are engineered to be coupled to the QP's qubits. Quadrupolar nuclear spins couple to the local electric field gradient and thus are sensitive to acoustic modulations [96] which enhance decoherence rates. An acoustic pulse can thus decohere the erasable register, while leaving the spin $\frac{1}{2}$ nuclei in the QP in any given state. The erasable register must then be repolarized. This use of erasable ancilla results in even small QCs being quite powerful since quantum resources need not to be borrowed for non-computational tasks.

\subsection{Requirements}

The five requirements of a physical quantum computer are explored below. We hope to convince the reader that the requisites for building a functional prototype quantum computer are within reach through an amalgamation of solid state NMR methods. At the same time we caution that the proposed QIP device will function on 10 through perhaps 30 qubits, which while making it by far the largest quantum processor to date (and possibly just beyond what can presently be simulated on a classical computer) the system will not be scalable. We believe however that it is only through the exploration of such quantum devices that scalable quantum computers might someday be realized. We consider each requirement in turn.

\subsubsection{Scalable mapping}

Although the correspondence between qubits and spin $\frac{1}{2}$ nuclei is clear in this case, its scaling is in fact limited by the need for having readily crystallizable molecules. In principle, adequate addressability of the qubits is available, if necessary by using quantum cellular automata implementation methods. We believe that it is possible to design appropriate molecules for up to perhaps 30 qubits. A more immediate obstacle to implementing this mapping is the presence of an internal Hamiltonian which does not preserve the standard basis for qubits, thus requiring active manipulation even for preserving the state. We address the issue of state preservation in the context of the requirement for implementing a sufficient set of gates.

The complexity of the internal Hamiltonian leads to two complications for viewing the spin system as a system of qubits. First, the eigenbasis is no longer known and so there is not a convenient and stable computational basis, and second the resonances are broadened by the many spin configurations of equal energy (configurational broadening). The second part is of little concern since we must fully control the internal Hamiltonian to have a QP, but the first point implies that individual qubits cannot be identified or addressed with RF pulses as easily as is the case in liquid state. The way out of the this difficulty is to realize that the flip-flop term does not commute with the difference of $\sigma_{z}$ operators, and that provided a spectral difference (such as that introduced by chemical shifts) can be made stronger than the dipolar couplings, the dynamics from the flip-flop term are effectively quenched by second averaging. Such a quenching is of course guaranteed in the case of heteronuclear coupling since the differences in Larmor frequencies are some $\mathrm{MHz}$ while the dipolar couplings at most in the tens of $\mathrm{kHz}$.

For the molecular system to be used in this proposal we need only concern ourselves with the spins that make up the individual QPs, since the deuterated bath will be decoupled from these, and each molecular QP will be well isolated from all other copies. The QP molecules should thus have the following properties:

1) Crystallize easily into large single crystals with only one molecule per unit cell.

2) Have high Debye temperatures $\left(>100^{\circ} \mathrm{K}\right)$.

3) Be dielectric.

4) Be composed of a few elements in addition to ${ }^{1} \mathrm{H}$ for which there is an isotope with no spin, and another with spin $\frac{1}{2}$ ( $\operatorname{such}$ as $\left.{ }^{13} C\right)$.

5) Have an orientation in which all spins are individually addressable.

The complete chemical shift tensors (with principal axis systems) are only known precisely for a few molecules since there has been little motivation to measure these. However, there are enough to ensure 
that finding a 10 qubit system with the above properties is only a modest challenge. We will start by exploring organic acids since they have been widely studied, and crystallize well. An illustrative example is malonic acid [47], which is shown in Fig. 9. An excellent area for appreciating the complexity of homonuclear dipolar coupled spins is zero field spectroscopy [135]. Zero-field spectroscopy removes the symmetry breaking introduced by the applied magnetic field and permits single crystal like spectra to be acquired for powdered

samples. Pines and coworkers have reported both experiments and simulations of small spin systems that show the power and complexity of these methods, and with coupling constants spanning a range of $60 \mathrm{kHz}$ for small organic molecules 135 .

\subsubsection{State preparation}

The state preparation, while perhaps appearing to be complex, is actually the simplest part of the protocol. We take advantage of the large Zeeman splitting of the electron spins so that we have a highly polarized quantum bath at liquid Helium temperatures. This saves us from having to work at the much more challenging $\mathrm{mK}$ temperatures. This polarization can be transferred to the deuterium nuclei by an Overhauser type effect through dynamic nuclear polarization, where a single microwave transition is saturated. The relatively short spin lattice relaxation time of the electrons coupled with their high photon energy provides a large effective heat capacity from which to cool the deuterons. In existing experiments, diamagnetic crystal polarizations of greater than $90 \%$ were achieved by such means [2]. An experiment more closely related to this proposal is De Boer's polarization of a deuterated sample of ethanediol (a sample containing ${ }^{13} C$, ${ }^{1} H$ and ${ }^{2} D$ nuclei). The polarization for ${ }^{1} H$ and ${ }^{2} D$ reached $80 \%$ and $30 \%$, respectively [29]. The cooling of the deuterium bath to sub-mK spin temperatures is a preparation step that is done before starting the computation. Following this the electrons will no longer take part in any aspect of the computation and the deuterium will act as a polarized bath of nearly infinite heat capacity that the qubits of the QP can be brought into contact at will. Connection to the polarized deuterium bath will be accomplished through Hartmann-Hahn cross polarization transfer [50], where two nuclear species are put into an interaction frame in which their apparent Zeeman energies are matched.

Since the QCs are only very dilute in the deuterium bath, the heat capacity of the bath will be much greater than that of the $\mathrm{QC}$ meaning that the final polarization of the $\mathrm{QC}$ and bath (following crosspolarization) will be very nearly the initial polarization of the bath and the bath may be in contact repeatedly without a need to refresh it from the electrons. Of course the suggestion that the bath may be reused implies that as we decouple it we must do so in such a way as to preserve its polarization.

Finally, although the QC has been prepared in a state that is quite close to a pure state, we will still need to rely on pseudo-pure or state purification schemes 104, perhaps in conjunction with the resettable register, to achieve a reliable pure state.

\subsubsection{Gates}

The internal Hamiltonian in conjunction with the coupling to RF fields provide all of the components necessary to create, in principle, any gate. Under the assumption that the resonance frequencies of the spins are sufficiently far apart, single qubit gates can be implemented by selective RF pulses, while two-qubit gates are implemented by delays which exploit the connected coupling network of the internal Hamiltonian. Appropriate refocusing schemes are required to eliminate unwanted contributions from the internal Hamiltonian. In particular, there must be a means for suppressing the internal Hamiltonian completely. If we can suspend the evolution of the internal motions, then we can always add back in any parts we desire. In earlier work we and others have devised time-suspension sequences that suppress the internal Hamiltonian to any desired precision [27] and have shown how to add back desired pieces of it 86]. The complications introduced here are that the gates should never interfere with the procedure for ${ }^{2} D$ decoupling of the bath from the QP. This requires synchronizing the various multiple-pulse cycles and designing them from the start to work together.

\subsubsection{Noise}

Decoherence times of dielectric crystals below their Debye temperature are notoriously long in NMR since there is no spontaneous emission and the phonon spectrum is very sparse. The only relaxation pathway is via defects, and thus will be very sample dependent. The relaxation rate $1 / T_{1}$ goes as $\left(H_{e} / H_{0}\right)\left(1 / T_{1 e}\right)\left(1-p^{2}\right)$ 
where $H_{e}$ is the field due to the electrons, $H_{0}$ is the applied magnetic field, $T_{1 e}$ is the electron relaxation time and $p$ the electron polarization. So in this approximation, as the electrons become polarized, the nuclear relaxation rate vanishes 45 .

In a system of dilute molecules with the spin transport through the lattice turned off through multiplepulse decoupling, we expect that the decoherence times will be even longer than those typically observed for concentrated samples of abundant spins 81]. Times on the order of hours are common (malonic acid crystals have a $T_{1}$ of order one hour) and for clean cubic systems there are examples of relaxation times being so long as to be unmeasurable. Even given relatively slow clock speeds in the ms, it is clear that spin lattice relaxation will not significantly limit the computational complexity of the proposed quantum computer.

Of course there is the related issue of the spin-spin relaxation time. Any difference between the spin-spin and the spin-lattice relaxation times are due to spin-spin interactions within the QP and these interactions must be precisely controlled to have a functional quantum computer. This puts a premium on our ability to apply average Hamiltonian theory, with multiple pulse cycles to take full control of the spin system, but this is exactly where NMR spectroscopy excels.

\subsubsection{Measurement}

The measurement process does not significantly differ from that used in liquid state NMR. The sample is still an ensemble of processors, and though the polarization is higher and the sample much more dilute, there will still be a sufficient number of spins to have good statistics.

\section{Future generations QIP based on engineered control of spin systems}

Although we hope to reach quite complex Hilbert spaces with the solid state NMR approach to QIP, this still does not yield a scalable quantum computer. The number of qubits is limited by the addressing scheme, the gates are far too slow, and the cost of additional qubits too high. While the progression towards more complex QIPs is stepwise, it is useful to have a trajectory in mind for a path to a scalable quantum computer.

Keeping to our model of a QP as a quantum simulation run on a physical system, there are two general approaches to implement the required dynamics:

1) Use the internal Hamiltonian and limited external control and move to an appropriate interaction frame to enable qubit-specific operations. This approach is limited as we can only scale down part of the Hamiltonian.

2) Modify the local Hamiltonian by near field interactions that are qubit-selective based on their spatial position and extent.

It appears that the second (or engineered) approach will be the eventual direction, but control that relies on the internal Hamiltonian is what can be done with high fidelity today. Although there have been some steps taken towards engineered systems [58, 57, 125, little is known about how to manufacture such devices, and even less about how to control them.

As the field progresses towards more complex QIP it appears that there are three regimes of complexity/decoherence:

1) Small scale QPs: Fully coherent control over a small quantum system, decoherence is not a significant problem and the dynamics could be simulated on a work station - this is where we are today.

2) Medium scale QPs: Complex and high fidelity coherent control of a modest quantum system. Decoherence is present but we can demonstrate accessibility of the full Hilbert space. The dynamics are no longer easily simulated on classical computers - this is where solid state NMR QIP is headed.

3) Large scale QPs: Local near field control of QPs' components making general transformations of many qubits much more accessible. The effects of decoherence on these systems is much more significant requiring sophisticated error-control schemes. 
The proposed solid state NMR device is intended to be medium scale, allowing exploration of problems not classically accessible while avoiding the decoherence problems of the more scalable near-field devices.

\section{Conclusion}

By enabling reasonably reliable manipulations of quantum information, NMR offers a useful test bed for QIP. The elements essential [33] for quantum information have been demonstrated. It is also moving theoretical successes from the desk to the laboratory benchtop and helps in understanding the difficulties of harnessing the power of quantum computation. NMR techniques have been developed in the spectroscopy community to deal with a wide range of coherent errors and although the final technology for quantum computation might not resemble what we can imagine today, the set of techniques being developed are needed to make progress.

Another important contribution from NMR has been to question some of the naive explanations of the power of quantum computation such as the need for pure states and entanglement. As a result, it has been realized that QIP extends well beyond quantum computing or communication, and the relative computational power of devices which do not fit the standard model is starting to be explored.

Despite the limitations of liquid state NMR, it is likely to be the first technology to control ten qubits. Many of these limitations will be overcome by a second generation of NMR based devices which has the potential to exceed the classical predictable realm. Our solid state proposal, by starting with high polarization and having a resettable register, allows a better understanding of practical quantum error correction. It is our hope that it will also allow a better understanding of quantum simulation and decoherence. As QIP devices access increasing numbers of qubits, it becomes increasingly difficult to simulate them using classical computers (and is impossible at around 30-40 qubits). This should be seen as an opportunity to investigate a new territory inaccessible to classical simulations. Systematic ways to deal with the complexity of these systems will have to be developed such as benchmarking and trouble shooting imperfections of physical devices. On the other hand the number of qubits available in solid state NMR is still small compared to what is needed for implementing the gamut of fault tolerant techniques. We will thus have to develop methods to investigate extremely large Hilbert spaces and verify the quality of the implemented operations. The work in NMR will contribute to these developments.

The recent discovery that quantum mechanics allows more powerful manipulations of information than its classical counterpart has the potential to revolutionize information processing. Although the accuracy threshold theorem shows that in principle, the limitations of quantum systems due to noise and decoherence can be overcome, this does not mean that the road to scalable QIP will be without difficulties. The required accuracies will be hard to achieve in practice, and at present we can only explore small scale quantum information.

It is of course possible that we will not be able to reach the accuracy threshold nor ever implement algorithmically interesting quantum computations. However, as more than 100 spin coherences have been manipulated, controlled and observed in solid state NMR, the main concern is our ability to maintain information in effectively distinguishable qubits. This is both a question of complexity and of stability. So while we are still struggling with the new quantum technology and its control, we are approaching the situation where we can ask whether there is any new physics which prohibits scalable manipulations of quantum information. As a result, the significance to practical and fundamental physics promises to be profound.

Acknowledgements. This work was suppported by the U.S. Army Research Office, DARPA and the National Security Agency. DGC thanks the Center for Non-linear Systems at Los Alamos National Laboratory for its hospitality.

\section{References}

[1] A. Abragam. Principles of Nuclear Magnetism. Clarendon Press, Oxford, England, 1961.

[2] A. Abragam and M. Goldman. Nuclear Magnetism: Order and Disorder. Oxford Scientific Pub., New York, 1982. 
[3] D. Aharonov and M. Ben-Or. Fault-tolerant quantum computation with constant error. In Proceedings of the 29th Annual ACM Symposium on the Theory of Computation (STOC), pages 176-188, New York, New York, 1996. ACM Press.

[4] A. Ambainis, L. J. Schulman, and U. Vazirani. Computing with highly mixed states. quant-ph/0001066, 2000 .

[5] A. G. Anderson and E. L. Hahn. Spin echo storage technique. US Patent \# 2,714,714, 1955.

[6] A.G. Anderson, R. Garwin, E. L. Hahn, J. W. Horton, and G. L. Tucker. Spin echo serial storage memory. J. App. Phys., 26:1324-1338, 1955.

[7] E.R. Andrew, A. Bradbury, and R.G. Eades. Removal of dipolar broadening of Nuclear Magnetic Resonance spectra of solids by specimen rotation. Nature, 183:1802-1803, 1959.

[8] W. P. Aue, E. Bartholdi, and R. R. Ernst. 2-dimensional spectroscopy: Application to Nuclear Magnetic Resonance. J. Mag. Res., 64:2226-2229, 1976.

[9] A. Barenco, A. Ekert, K.-A. Suominen, and P. Törmä. Approximate quantum Fourier transform and decoherence. Phys. Rev. A, 54:139-146, 1996.

[10] J. Baum, M. Munowita, A. Garroway, and A. Pines. Multiple-quantum dynamics in solid-state NMR. J. Chem. Phys., 83:2015-2125, 1985.

[11] A. Bax, R. Freeman, and T. A. Frenkel. NMR technique for tracing out the carbon skeleton of an organic molecule. J. Am. Chem. Soc., 1103:2102-2104, 1981.

[12] C. H. Bennett and D. P. DiVincenzo. Quantum computing: Towards an engineering era. Nature, 377:389-390, 1995.

[13] C.H. Bennett and P.W. Shor. Quantum information theory. IEEE Trans. Inf. Theory, 44:2724-2742, 1998.

[14] F. Bloch. Nuclear induction. Phys. Rev., 70:460-485, 1946.

[15] N. Bloembergen. On the interaction of nuclear spins in a crystalline lattice. Physica, 15:386-426, 1949.

[16] N. Bloembergen and R. V. Pound. Radiation damping in magnetic resonance. Phys. Rev., 95:263-267, 1954.

[17] G. Bodenhausen, H. Kogler, and R. R. Ernst. Selection of coherence-transfer pathways in NMR pulse experiments. J. Mag. Res., 58:370-388, 1984.

[18] D.P. Burum and R.R. Ernst. Net polarization transfer via a J-ordered state for signal enhancement of low-sensitivity nuclei. J. Mag. Res., 39:163-168, 1980.

[19] G. C. Chingas, A. N. Garroway, R. D. Bertrand, and W. B. Moniz. Zero quantum NMR in the rotating frame: J-cross polarization in AXN systems. J. Chem. Phys., 74:127-156, 1981.

[20] I. L. Chuang, N. Gershenfeld, and M. Kubinec. Experimental implementation of fast quantum searching. Phys. Rev. Lett., 80:3408-3411, 1998.

[21] I. L. Chuang, N. Gershenfeld, M. Kubinec, and D. Leung. Bulk quantum computation with Nuclear Magnetic Resonance: Theory and experiments. Proc. R. Soc. Lond. A, 454:447-467, 1998.

[22] I. L. Chuang, L. M. K. Vandersypen, X. Zhou, D. W. Leung, and S. Lloyd. Experimental realization of a quantum algorithm. Nature, 393:143-146, 1998.

[23] D. Collins, K. W. Kim, W. C. Holton, H. Sierzputowska-Gracz, and E. O. Stejskal. NMR quantum computation with indirectly coupled gates. quant-ph/9910006, 1999. 
[24] D. Cory, A.E. Dunlop, T.F. Havel, S.S. Somaroo, and W. Zhang. The effective Hamiltonian of the Pound-Overhauser control-NOT gate. quant-ph/9809045, 1998.

[25] D. G. Cory, A. F. Fahmy, and T. F. Havel. Nuclear Magnetic Resonance spectroscopy: An experimentally accessible paradigm for quantum computing. In T. Toffoli et al., editor, Proceedings of the 4th Workshop on Physics and Computation, pages 87-91, Boston, Massachusetts, 1996. New England Complex Systems Institute.

[26] D. G. Cory, W. Maas, M. Price, E. Knill, R. Laflamme, W. H. Zurek, T. F. Havel, and S. S. Somaroo. Experimental quantum error correction. Phys. Rev. Lett., 81:2152-2155, 1998.

[27] D. G. Cory, J. B. Miller, and A. N. Garroway. Time-suspension multiple-pulse sequences : Applications to solid-state imaging. J. Mag. Res., 90:205-213, 1990.

[28] D. G. Cory, M. D. Price, and T. F. Havel. Nuclear Magnetic Resonance spectroscopy: An experimentally accessible paradigm for quantum computing. Physica D, 120:82-101, 1998.

[29] W. DeBoer, M. Borghini, K. Morimoto, and T. Niinikoski. Dynamic polarization of protons, deuterons, and carbon-13 nuclei: Thermal contact between nuclear spins and an electron spin-spin interaction reservoir. J. Low Temp. Phys., 15:249-267, 1974.

[30] D. P. DiVincenzo. Real and realistic quantum computers. Nature, 393:113-115, 1998.

[31] D.P. DiVincenzo. Quantum computation. Science, 270:255-261, 1995.

[32] D.P. DiVincenzo. Two-bit gates are universal for quantum computation. Phys. Rev. A, 51:1015-1022, 1995.

[33] D.P. DiVincenzo. The physical implementation of quantum computation. To appear in Fort. Phys., special issue on "Experimental Proposals for Quantum Computation". quant-ph/0002077, 2000.

[34] D. M. Doddrell, D. T. Pegg, and M. R. Bendall. Distortion enhancement of NMR signals by polarization transfer. J. Mag. Res., 48:323-327, 1982.

[35] K. Dorai, Arvind, and A. Kumar. Implementing quantum logic operations, pseudo-pure states and the Deutsch-Jozsa algorithm using non-commuting selective pulses in NMR. Phys. Rev. A, 61:042306/1-7, 2000 .

[36] L. Emsley and A. Pines. Lectures on pulsed NMR. In B. Maraviglia, editor, Proceedings of the International School of Physics "Enrico Fermi", volume CXXIII, pages 2949-2954, 1994.

[37] R. R. Ernst, G. Bodenhausen, and A. Wokaun. Principles of Nuclear Magnetic Resonance in One and Two Dimensions. Oxford University Press, Oxford, 1994.

[38] X. Fang, X. Zhu, M. Feng, and F. Du X. Mao. Experimental implementaton of dense coding using Nuclear Magnetic Resonance. Phys. Rev. A, 61:022307/1-5, 1999.

[39] S. Forsen and R. Hoffman. J. Mag. Res., 29:433, 1963.

[40] L. Fu, J. Luo, L. Xiao, and X. Zeng. Experimental realization of discrete Fourier transformation on NMR quantum computer. quant-ph/9905083, 1999.

[41] N. A. Gershenfeld and I. L. Chuang. Bulk spin resonance quantum computation. Science, 275:350-356, 1997.

[42] R. Ghose, T.R. Eyken, and G. Bodenhausen. Average Liouvillian theory revisited: Cross-correlated relaxation between chemical shift anisotropy and dipolar couplings in the rotating frame in Nuclear Magnetic Resonance. Mol. Phys., 96:1281-1288, 1999. 
[43] S. J. Glaser, T. Schulte-Herbrüggen, M. Sieveking, O. Schedletzky, N. C. Nielsen, O. W. Sörensen, and C. Griisigner. Unitary control in quantum ensembles: Maximizing signal intensity in coherent spectroscopy. Science, 280:421-425, 1998.

[44] M. Goldman. Spin Temperature and Magnetic Resonance in Solids. Oxford Univ. Press, Oxford, 1970.

[45] T. Gunter and C. Jeffries. Proton spin-lattice relaxation in (Nd, $\mathrm{La})_{2} \mathrm{Mg}_{3}\left(\mathrm{NO}_{3}\right)_{12} \cdot 24 \mathrm{H}_{2} \mathrm{O}$ in high fields and low temperatures. Phys. Rev., 159:290-295, 1967.

[46] U. Haeberlen. High Resolution NMR in Solids: Selective Averaging. Academic Press, New York, USA, 1976.

[47] U. Haeberlen. Multiple-pulse techniques in solid state NMR. Mag. Res. Rev., 10:81-110, 1985.

[48] U. Haeberlen and J. S. Waugh. Coherent averaging effect in magnetic resonance. Phys. Rev., 175:453$467,1968$.

[49] E. L. Hanh. Spin echoes. Phys. Rev., 80:580-594, 1950.

[50] S.R. Hartman and E.L. Hahn. Nuclear double resonance in the rotating frame. Phys. Rev., 128:20422053, 1962.

[51] J. Jeener. Superoperators in magnetic resonance. Adv. Mag. Res., 10:2-51, 1982.

[52] C. D. Jeffries. Dynamic Nuclear Orientation. Wiley, New York, USA, 1963.

[53] J. A. Jones and M. Mosca. Implementation of a quantum algorithm to solve Deutsch's problem on a Nuclear Magnetic Resonance quantum computer. J. Chem. Phys., 109:1648-1653, 1998.

[54] J. A. Jones, M. Mosca, and R. H. Hansen. Implementation of a quantum search algorithm on a quantum computer. Nature, 392:344-346, 1998.

[55] J. A. Jones, V. Vedral, A. Ekert, and G. Castagnoli. Geometric quantum computation with NMR. Nature, pages 869-871, 2000.

[56] J.A. Jones. NMR quantum computation: A critical evaluation. To appear in Fort. Phys., special issue on "Experimental Proposals for Quantum Computation". quant-ph/0002085, 2000.

[57] B. E. Kane. A silicon-based nuclear spin quantum computer. Nature, 393:133-137, 1998.

[58] J.M. Kikkawa and D.D. Awschalom. All-optical magnetic resonance in semi-conductors. Science, $287: 473-476,2000$.

[59] A. Yu. Kitaev. Quantum computations: algorithms and error correction. Uspekhi Mat. Nauk., 52:53$112,1997$.

[60] E. Knill. Approximation by quantum circuits. Technical Report LAUR-95-2225, Los Alamos National Laboratory, knilllanl.gov, 1995. quant-ph/9508006.

[61] E. Knill, I. Chuang, and R. Laflamme. Effective pure states for bulk quantum computation. Phys. Rev. A, 57:3348-3363, 1998.

[62] E. Knill and R. Laflamme. On the power of one bit of quantum information. Phys. Rev. Lett., 81:5672-5675, 1998.

[63] E. Knill and R. Laflamme. Quantum computation and quadratically signed weight enumerators. quantph/9909094, 1999.

[64] E. Knill, R. Laflamme, R. Martinez, and C.-H. Tseng. An algorithmic benchmark for quantum information processing. Nature, 404:368-370, 2000. 
[65] E. Knill, R. Laflamme, and L. Viola. Theory of quantum error correction for general noise. Phys. Rev. Lett., 84:2525-2528, 2000.

[66] E. Knill, R. Laflamme, and W. H. Zurek. Resilient quantum computation. Science, 279:342-345, 1998.

[67] R. Laflamme, E. Knill, W. H. Zurek, P. Catasti, and S. V. S. Mariappan. NMR Greenberger-HorneZeilinger states. Phil. Trans. R. Soc. Lond. A, 356:1941-1948, 1997.

[68] R. Landauer. Is quantum mechanically coherent computation useful? Phil. Trans. R. Soc. Lond. A, 353:367-376, 1995.

[69] D. Leung, L. Vandersypen, X. L. Zhou, M. Sherwood, C. Yannoni, M. Kubinec, and I. Chuang. Experimental realization of a two-bit phase damping quantum code. Phys. Rev. A, 60:1924-1943, 1999.

[70] M. H. Levitt and R. Freeman. Composite pulse decoupling. J. Mag. Res., 43:502-507, 1981.

[71] D. A. Lidar, I. L. Chuang, and K. B. Whaley. Decoherence-free subspaces for quantum computation. Phys. Rev. Lett., 81:2594-2597, 1998.

[72] N. Linden, H. Barjat, and R. Freeman. An implementation of the Deutsch-Jozsa algorithm on a three-qubit NMR quantum computer. Chem. Phys. Lett, 296:61, 1998.

[73] N. Linden and R. Freeman E. Kupce. NMR quantum logic gates for homonuclear spin systems. Chem. Phys. Lett., 311:321-327, 1999.

[74] S. Lloyd. A potentially realizable quantum computer. Science, 261:1569-1571, 1993.

[75] S. Lloyd. Almost any quantum logic gate is universal. Phys. Rev. Lett., 75:346-349, 1996.

[76] S. Lloyd. Quantum mechanical computers. Science, 273:140-145, 1996.

[77] W. Magnus. Comm. Pure Appl. Math., 7:649, 1954.

[78] P. Mansfield and P. Morris. NMR imaging in medicine. Adv. Mag. Res., S2:1-343, 1982.

[79] M. Marjanska, I.L. Chuang, and M.G. Kubinec. Demonstration of quantum logic gates in liquid crystal Nuclear Magnetic Resonance. J. Chem. Phys., 112:5095-5099, 2000.

[80] R. Marx, A. F. Fahmy, J. M. Myers, W. Bermel, and S. J. Glaser. Realization of a 5-bit NMR quantum computer using a new molecular architecture. quant-ph/9905087, 1999.

[81] I. Mavlonazarov, V.M. Mikushev, and E.V. Charnaya. Direct measurement of the lattice and impurity components of nuclear spin-lattice relaxation under magnetic-saturation conditions. JETP Lett., 56:13$16,1992$.

[82] G. A. Morris and R. Freeman. Enhancement of Nuclear Magnetic Resonance signals by polarization transfer. J. Am. Chem. Soc., 101:760-762, 1979.

[83] G.A. Morris and R. Freeman. Selective excitation in Fourier transform Nuclear Magnetic Resonance. J. Mag. Res., 29:433-462, 1978.

[84] K. T. Mueller, B. Q. Sun, G. C. Chingas, J. W. Zwanziger, T. Terao, and A. Pines. Dynamic-angle spinning of quadrupolar nuclei. J. Mag. Res., 86:470-487, 1990.

[85] A. Munowitz and A. Pines. Principles and applications of multiple-quantum NMR. Adv. Chem. Phys., $66: 1-152,1987$.

[86] M. Munowitz. Coherence and NMR. J. Wiley and Sons, New York, 1988.

[87] G. Navon, T. Room Y. Q. Song, R. E. Taylor A. Appelt, and A. Pines. Enhancement of solution NMR and MRI with laser-polarized xenon. Science, 271:1848-1851, 1996. 
[88] R. Nelson, S. Lloyd, and D. G. Cory. Experimental demonstration of Greenberger-Horne-Zeilinger correlations using Nuclear Magnetic Resonance. Phys. Rev. A, 61:022106/1-5, 2000.

[89] M. A. Nielsen, E. Knill, and R. Laflamme. Complete quantum teleportation. Nature, 396:52-55, 1998.

[90] K. J. Packer and K. M. Wright. The use of single-spin operator basis-sets in the NMR spectroscopy of scalar-coupled spin systems. Mol. Phys., 50:797-813, 1983.

[91] S. Parker and M.B. Plenio. Efficient factorization with a single pure qubit. quant-ph/0001066, 2000.

[92] A. Pines, D. J. Ruben, S. Vega, and M. Mehring. New approach to high-resolution proton NMR in solids: Deuterium spin decoupling by multiple-quantum transitions. Phys. Rev. Lett., 36:110-113, 1976.

[93] J. Preskill. Reliable quantum computers. Proc. R. Soc. Lond. A, 454:385-410, 1998.

[94] M. D. Price, S. S. Somaroo, A. E. Dunlop, T. F. Havel, and D. G. Cory. Generalized methods for the development of quantum logic gates for an NMR quantum information processor. Phys. Rev. A, 60:2777-2780, 1999.

[95] M. D. Price, S. S. Somaroo, C.-H. Tseng, J. C. Gore, A. F. Fahmy, T. F. Havel, and D. G. Cory. Construction and implementation of NMR quantum logic gates for two spin systems. J. Mag. Res., 140:371-378, 1999.

[96] W. G. Proctor and W. H. Tanttila. Saturation of nuclear electric quadrupole energy levels by ultrasonic excitation. Phys. Rev., 98:1854-1854, 1955.

[97] E. M. Purcell, H. C. Torrey, and R. V. Pound. Resonance absorption by nuclear magnetic moments in a solid. Phys. Rev., 69:37-38, 1946.

[98] A. G. Redfield, S. D. Kunz, and E. K. Ralph. Resonance absorption by nuclear magnetic moments in a solid. J. Mag. Res., 19:114-117, 1975.

[99] A.G. Redfield. On the theory of relaxation processes. IBM J. Res. Dev., 1:19, 1957.

[100] A.G. Redfield. The theory of relaxation processes. Adv. Mag. Res., 1:1-32, 1965.

[101] W.-K. Rhim, A. Pines, and J. S. Waugh. Violation of the spin temperature hypothesis. Phys. Rev. Lett., 25:218-220, 1970.

[102] A. Saupe. Das Protonenresonanzenspektrum von orientiertem Benzol in nematisch-kristallinflüssiger Lösung. Zeit. Naturforschung A, 20:572-580, 1965.

[103] C.P. Schlichter. Principles of Magnetic Resonance. Harper \& Row, New York, 1963.

[104] L. J. Schulman and U. Vazirani. Scalable NMR quantum computation. In Proceedings of the 31th Annual ACM Symposium on the Theory of Computation (STOC), pages 322-329, El Paso, Texas, 1998. ACM Press.

[105] B. Schumacher. Sending entanglement through noisy quantum channels. Phys. Rev. A, 54:2614-2628, 1996.

[106] P. W. Shor. Scheme for reducing decoherence in quantum computer memory. Phys. Rev. A, 52:24932496, 1995.

[107] P. W. Shor. Polynomial-time algorithms for prime factorization and discrete logarithms on a quantum computer. SIAM J. Comput., 26:1484-1509, 1997.

[108] L. C. Snyder and S. Meiboom. Theory of proton NMR with deuteron decoupling in nematic liquid crystalline solvents. J. Chem. Phys., 58:5096-5103, 1973. 
[109] A. Sodickson and D.G. Cory. A generalized $k$-space formalism for treating the spatial aspects of a variety of NMR experiments. Prog. Nucl. Mag. Res. Spect., 33:77-108, 1998.

[110] S. Somaroo, C.-H. Tseng, T. F. Havel, R. Laflamme, and D. G. Cory. Quantum simulations on a quantum computer. Phys. Rev. Lett., 82:5381-5384, 1999.

[111] S. S. Somaroo, D. G. Cory, and T. F. Havel. Expressing the operations of quantum computing in multiparticle geometric algebra. Phys. Lett. A, 240:1-7, 1998.

[112] O. W. Sörensen. Polarization transfer experiments in high-resolution NMR spectroscopy. Prog. Nucl. Mag. Res. Spect., 21:503-569, 1989.

[113] O. W. Sörensen. A universal bound on spin dynamics. J. Mag. Res., 86:435-440, 1990.

[114] O. W. Sörensen, G. W. Eich, M. H. Levitt, G. Bodenhausen, and R. R. Ernst. Product operatorformalism for the description of NMR pulse experiments. Prog. Nucl. Mag. Res. Spect., 16:163-192, 1983.

[115] A. Steane. Multiple particle interference and quantum error correction. Proc. R. Soc. Lond. A, 452:2551-2577, 1996 .

[116] A. Steane. Quantum computing. Rep. Progr. Phys., 61:117-173, 1998.

[117] M. E. Stoll, A. J. Vega, and R .W. Vaughan. Explicit demonstration of spinor character for a spin-1/2 nucleus using NMR interferometry. Phys. Rev. A, 16:1521-1524, 1977.

[118] D. Suter, A. Pines, and M. Mehring. Indirect phase detection of NMR spinor transitions. Phys. Rev. Lett., 57:242-244, 1986.

[119] G. Teklemarian, Y. Sharf, C.-H. Tseng, T. F. Havel, and D. G. Cory. An NMR analog of the quantum entanglement eraser. Submitted to Phys. Rev. Lett., 2000.

[120] C.-H. Tseng, S. Somaroo, Y. Sharf, E. Knill, R. Laflamme, T. F. Havel, and D. G. Cory. Quantum simulation of a three-body interaction Hamiltonian on an NMR quantum computer. Phys. Rev. A, 61:012302/1-6, 2000 .

[121] W. G. Unruh. Maintaining coherence in quantum computers. Phys. Rev. A, 51:992-997, 1995.

[122] F.J.M. Vandeven and G.W. Hilbers. A simple formalism for the description of multiple-pulse experiments: Application to a weakly coupled 2-spin (I=1/2) system. J. Mag. Res., 54:512-520, 1983.

[123] L. Viola, E. Knill, and S. Lloyd. Dynamical decoupling of open quantum systems. Phys. Rev. Lett., 82:2417-2421, 1999.

[124] L. Viola and S. Lloyd. Dynamical suppression of decoherence in two-state quantum systems. Phys. Rev. A, 58:2733-2744, 1998.

[125] R. Vrijen, E. Yablonovitch, K. Wang, H. W. Jiang, A. Balandin, V. Roychowdhury, T. Mor, and D. DiVincenzo. Electron spin resonance transistors for quantum computing in silicon-germanium heterostructures. quant-ph/9905096, 2000.

[126] W. S. Warren, D. P. Weitekamp, and A. Pines. Theory of selective excitation of multiple-quantum transitions. J. Chem. Phys., 73:2084-2099, 1980.

[127] W.S. Warren. Selective excitation of multiple quantum coherence in Nuclear Magnetic Resonance. Phys. Rev. Lett., 43:1791-1794, 1979.

[128] J. S. Waugh. Theory of broad-band spin decoupling. J. Mag. Res., 50:30-49, 1982.

[129] J. S. Waugh, L. M. Huber, and U. Haeberlen. Approach to high resolution NMR in solids. Phys. Rev. Lett., 20:180-182, 1968. 
[130] Y. S. Weinstein, S. Lloyd, and D. G. Cory. Implementation of the quantum Fourier transform. quant ph/9906059, 1999.

[131] R. M. Wilcox. Exponential operators and parameter differentiation in quantum physics. J. Math. Phys., 8:962-982, 1967.

[132] R.A. Wind, M.J. Duijvestijn, C. Vanderlugt, A. Manenschijn, and J. Vriend. Applications of dynamic nuclear-polarization in ${ }^{13} \mathrm{C}$ NMR in solids. Prog. Nucl. Mag. Res. Spect., 17:33-67, 1985.

[133] C. S. Yannoni, M.H. Sherwood, L.M.K. Vandersypen, D.C. Miller, M.G. Kubinec, and I. Chuang. Nuclear Magnetic Resonance quantum computing using liquid crystal solvents. Appl. Phys. Lett., 75:3563-3565, 1999.

[134] P. Zanardi and M. Rasetti. Noiseless quantum codes. Phys. Rev. Lett., 79:3306-3309, 1997.

[135] D. Zax, A. Bielicki, K. Zilm, A. Pines, and D. Weitekamp. Zero-field NMR and NQR. J. Chem. Phys., 83:4877-4905, 1985.

[136] W. Zhang and D. G. Cory. First direct measurement of the spin diffusion rate in a homogenous solid. Phys. Rev. Lett., 80:1324-1327, 1998.

[137] X. Zhou, D. W. Leung, and I. L. Chuang. Quantum algorithms which accept hot qubit inputs. quant$\mathrm{ph} / 9906112,1999$.

[138] W. H. Zurek. Decoherence and the transition from quantum to classical. Phys. Today, 44:36-44, 1991. 